\title{
IMPACT OF GROUND MOTION DURATION AND SOIL NON- LINEARITY ON THE SEISMIC PERFORMANCE OF SINGLE PILES
}

\author{
Alessandro Tombari [Corresponding author] \\ School of Environment and Technology, University of Brighton, Cockcroft Building, Lewes Road, Brighton, BN2 4GJ \\ United Kingdom. a.tombari@brighton.ac.uk \\ M. Hesham El Naggar \\ Department of Civil and Environmental Engineering, Faculty of Engineering, Western University, London, Canada. \\ naggar@uwo.ca \\ Francesca Dezi \\ DESD, University of the Republic of San Marino, Via Salita alla Rocca 44, 47890, San Marino, Republic of San Marino \\ francesca.dezi@tin.itunirsm.sm
}

Keywords: Soil-pile interaction, BNWF model; Incremental Dynamic Analyses; Soil degradation;

Soil hardening; Soil cave-in; Ground Motion Duration; Seismic Performance.

\section{ABSTRACT:}

Pile foundations strongly influence the performance of supported structures and bridges during an earthquake. In case of strong earthquake ground motion, soft soils may be subjected to large deformation manifesting aspects typical of the non-linear behaviour such as material yielding, gapping and cyclic degradation-. Therefore, nonlinear soil-pile interaction models should be able to capture these effects and improve the prediction of the actual seismic loading transferred from the foundation to the superstructure. In this paper, a beam on nonlinear Winkler foundation (BNWF) model is used, which can simulate cyclic soil degradation/hardening, soil and structural yielding, slack zone development and radiation damping. Incremental Dynamic Analyses (IDAs) are performed to evaluate the effects of Ground Motion Duration (GMD) and soil non-linearity on the performance of single fixed-head floating piles. Various homogeneous and bilayer soil profiles are considered, including saturated clay and sand in either fully dry or saturated state and with different levels of compaction. In order to evaluate the effect of nonlinearity on the response, the results of the nonlinear analyses are compared with those obtained from linear soil-pile analysis in terms of bending moment envelope. Results show the relevance of considering the GMD on the 
performance of the single pile especially when founded on saturated soils. For low intensities and dry sandy soils, the linear soil-pile interaction model can be used for obtaining reliable results.

\section{INTRODUCTION}

The prediction of the performance of pile foundations during earthquakes is a fundamental task for the seismic design of structures and bridges. Most modern seismic codes (e.g. ASCE 41-06, ASCE 7-10, Eurocode 8, Italian technical code NTC, 2008, Mexico City Building Code, ASCE 41-06, ASCE 7-10) recommend accounting for soil-structure interaction effects in the seismic design of both foundations and superstructures. The mechanism of soil-pile-structure interaction has been extensively investigated by numerous researchers and methods for assessment of the seismic performance of soil-structure systems have improved significantly; a comprehensive review of the problem can be found in Kausel [1]. It is worth mentioning that most of the cited studies on soilstructure interaction are restricted to linearly elastic system by assuming that the relative displacement between foundation and soil remains small in the case of medium dense or firm ground when subjected to moderate earthquake motions. Therefore, when the soil behaves as a linear or equivalent-linear material, the entire soil-foundation-structure system can be subdivided into two separate sub domains, i.e. the superstructure and the soil-foundation, in order to apply the subdomain method [2-3] by separating the effects of inertial and kinematic interactions. On the other hand, when the ground is loose or soft or when the ground undergoes strong earthquake motions, those relative displacements become large and soil non-linearity becomes predominant, hence modifying considerably the dynamic response of the entire system. Converse to the solution based on elastic theory, nonlinear soil response entails gap formation, foundation uplifting, soil yielding as well as softening and hardening constitutive behaviours. Effects of the soil nonlinearity on structures with shallow embedded and deep foundations were observed by Trombetta et al. [4] and Dashti-Boulanger et al. [5] by means of centrifuge testing, while Pitilakis et al. [6] and Maugeri [7], Biondi et al. [8], and Abate and Massimino [9],_nvestigated effects of soil in reduced-scale shaking table tests. On the other hand, as observed by Meymand [810], the vast 
majority of centrifuge and shaking table tests of structures supported by pile foundations were conducted for investigating the soil-pile seismic response in cohesionless soils with liquefaction potential whilst many cases of piles are founded on soil manifesting potential for cyclic strength degradation. Shaking table tests on pile foundations with different pile head conditions are conducted by Chidichimo et al.Moccia [911] while Durante et al. [1012] investigated the soil-pileinteraction of a single degree of freedom supported by single and group pile foundation. It is worth mentioning that in both works nonlinear soil effects are elicited by comparison the actual response with the expected pile response in linear elastic material. Direct effects of the soil nonlinear constitutive behaviour of pile foundations were observed by Meymand, [810] through large scale shaking table tests.

A fully nonlinear analysis should be performed to properly evaluate the response of soil-pile system during strong ground motion events, rather than equivalent linear procedures that provide soil stiffness and damping ratio corresponding to the earthquake induced level of shear strain. Full 3D nonlinear soil-pile-structure interaction analyses are generally carried out through the finite

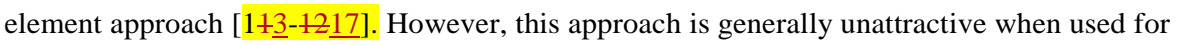
large nonlinear models.

An alternative approach consists of using the beam on nonlinear Winkler foundation (BNWF) model where the soil-pile interaction is simulated through nonlinear springs. The dynamic BNWF approach is a two-step method that uncouples the nonlinear behaviour of the near-field from the assumed linear or equivalent linear behaviour of the far-field in order to apply the principle of superposition of both effects. A few examples of different implementations can be found in 2127].

The dynamic soil-pile interaction effect is also referred to as kinematic interaction phenomenon, which is commonly incorporated in the framework of substructure technique. Nevertheless, kinematic interaction entails the stresses that are generated within the pile due to the propagation of the seismic waves through the soil excluding the inertial loading of the superstructure. In this 
context, the analysis of the kinematic interaction in nonlinear soil is an important task for the reliable assessment of the actual performance of foundation, as already investigated by Bentley and El Naggar (2000).[28]. Furthermore, the role of ground motion duration as an important factor the nonlinear structural response is still topic of some debate as evident from the different conclusions in [z229-2330].

In this paper, Incremental Dynamic Analyses (IDAs) are carried out in order to investigate the effects of the nonlinearity on the kinematic interaction of single piles. Although it is commonly used as tool to assess the capacity of structures [31], in this work, IDA is here performed to evaluate the performance of single piles by proposing a novel procedure to derive the scaled earthquake ground motions by means of an iterative site response analysis. A structural Intensity Measure (IM), namely the spectral acceleration, is used to scale the earthquake ground motions to multiple levels of intensity. This aims to generate results that might be easily extended to the structural counterpart so to create a link between geotechnical and structural engineering. The analysis combines the Allotey and El Naggar's BNWF model [23] with the new proposed framework for the equivalent linear site response analysis. Finally, the impact of the Ground Motion Duration (GMD) is evaluated by considering earthquake event with different duration scenarios.

\section{OBJEGTIVES METHODOLOGY AND SCOPE OF WORK}

This paper aims to evaluate the effects of Ground Motion Duration (GMD)-and soil non-linearity on the performance of single fixed-head floating piles. Incremental Dynamic Analyses (IDAs)-are performed by considering various homogeneous and bilayer soil profiles including: saturated clay and sand in either fully dry or saturated state, with different levels of compaction.

The non-linear kinematic interaction analysis proposed in this paper encompasses two steps. Firstly, the free-field displacements within the deposit along the pile are defined by means of a linearequivalent site response analysis starting from real accelerograms opportunely defined at the outcropping bedrock. Secondly, the soil-pile interaction is evaluated using a BNWF model, which 
approximates the soil-pile interaction using non-linear (p-y) springs in parallel with stiffness proportional dampers. This allows estimating the relative displacements between soil and pile due to the free-field motion. In the BNWF model the pile itself is modelled as a series of beam-column elements, each with discrete springs connecting the pile to the soil, and the free-field motion obtained within the deposit is applied to the p-y springs as excitation to the system.

In this paper, the BNWF model proposed by Allotey and El Naggar [1923] is employed for investigating the effect of soil nonlinearity on the seismic performance of a single pile. This BNWF model is able to simulate generalized dynamic normal force-displacement relationships, accounting for cyclic soil degradation/hardening, soil and structural yielding, slack zone development and radiation damping. In addition, this BNWF model was revised [z432] to account for the build-up of pore pressure due to cyclic loading and the different compaction levels of the soil in the vicinity of the pile in a simplified fashion. In particular, a generalized fatigue model based on the total stress method has been developed for simulating cyclic hardening/degradation of stiffness and/or strength that can account implicitly for several hysteretic soil-pile behaviours.

Finally, results demonstrating the effects of soil non-linearity and the duration of strong motion on the seismic behaviour of piles are compared to thaditionalthe results obtained by the linear soilpile analysisinteraction model defined by Dezi et al. [33-35].

\section{PILE-SOIL INTERACTION ANALYSIS WITH BNWF MODEL}

The lateral dynamic response of the pile $y(z, t)$ to the lateral dynamic load $p(z)$, which varies with depth $\mathrm{z}$, can be described by the general differential equation of motion for a beam on nonlinear Winkler foundation as follows:

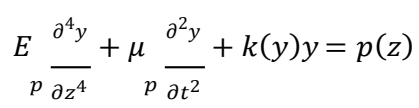

where $E_{p}$ is the Young's modulus of the pile, $\mu_{p}$ is the pile mass per unit length, and $k(y)$ is the nonlinear soil-pile reaction modulus. In this paper, $k(y)$ is described through the Allotey and El Naggar model [1923]. The dynamic BNWF model [1923] is a degrading polygonal hysteretic model that 
encompasses multilinear backbone curve with defined rules for loading, reloading and unloading. This model is able to capture the dynamic nonlinear behaviour of soil through the following features: i) it accounts for cyclic soil hysteresis through simulating unloading -reloading curves considering a set of rules such as those proposed by Pyke [2536]; ii) it can simulate gap formation and closing along the soil-pile interface for cohesive soils and reloading in the slack zone (by means of a strain-hardening curve) for cohesionless soils; iii) it can handle cyclic soil degradation/hardening due to the pore pressure changing and particle structure breakdown (changes in the clay microstructure and bond degradation); iv) finally, it accounts for radiation damping decreasing with the increases of soil non-linearity. The initial confining pressure at zero pile displacement is modelled as a pre-straining effect applied to the compression-only elements attached to both sides of the pile.

Therefore, due to its main features, this model is able to simulate synthesized generic cyclic normal force-displacement behaviour usually observed in sandy and clayey soil by setting proper model parameters having a physical significance owing to their derivation from standard field and laboratory tests. It simulates three main behaviours: the nonlinear monotonic force-displacement curve; the behaviour to unload-reload cycle; and the degradation or hardening under repeated cyclic loading. These features govern the global dynamic soil-pile interaction behaviour.

Generally, four different phenomenological models, as depicted in Figure 1, are discerned. In saturated soils (sand or soft clay), the cyclic response of the soil along the upper portion of pile is generally considered unconfined and is characterized by an inverted S-shaped hysteresis curve due to slack zone development (Figure 1.a). On the other hand, the cyclic response of soil along the lower segment of pile is considered confined and is characterized by an oval-shape hysteresis curve (Figure 1.b). In the case of dry soils (loose sand in particular), soil cave-in is expected to occur, hence the soil cyclic response is characterized by an oval-shape hysteresis curve along the upper portion of the pile as well. Undergoing cyclic loading, soils may exhibit both stiffness and strength degradation depending on the maximum strain amplitude and number of loading cycles experienced. For saturated soft clay, stiffness degradation 
is usually more significant than strength degradation [2637-2738], while for dry sands a typical response is expected (Figure 1.c). Finally, a brittle or softening behaviour characterizes the soil-pile response in stiff clay (Figure 1.d).

Backbone curves and cyclic behaviour

The backbone curve defined in this model is a multilinear curve defined by fitting a given (theoretical or experimental) non-linear force-displacement curve, namely a p-y curve. It is constituted by four segments that can either simulate monotonic (Figure 2a, segments 1, 2, 3 and 4) or post-peak behaviour (Figure 2a, segments $1,2,3^{*}$ and $4 *$ ). Each slope is determined by the ratio to the initial stiffness of the backbone curve defined by the parameter $K_{0}$. Cyclic behaviour is achieved by introducing specific unload-reload rules. Figure $2 \mathrm{~b}$ shows the unloading curve, termed the General Unloading Curve (GUC) (segments 5 - 6) and the reloading curve, termed the Standard Reload Curve (SRC) (segments 7 - 8 9 - 10), derived by scaling the backbone curve by a factor based on the force at the beginning of reloading or unloading [25 $\underline{36}$ ] and on the current cyclic degradation factor [z432], i.e.,

$$
\kappa=1 \pm \frac{p_{u r}}{\delta_{s} p_{f}}
$$

in which $p_{u r}$ is the current force at the onset of unloading or reloading, $\delta_{s}$ is the strength degradation parameter (described in more detail in the next paragraph) and $p_{f}$ is the ultimate force. The plus $(+)$ and minus (-) signs denote unloading and reloading, respectively. The model simulates either progressive gapping with depth and with the number of load cycles, or pile moving through the slack zone. The Direct Reload Curve (DRC) simulates the soil reaction when the pile moves through the slack zone and is designed as a convex strain-hardening curve controlled by a limiting-force parameter related to the past maximum force. Figure 2c depicts the formation of pure gap (segment 11), or slack zone (segment 12 characterized by a strain-hardening behaviour simulating the densification of the loose caved-in soil).

Cyclic degradation

A generalized fatigue formulation is implemented for simulating the cyclic soil hardening/degradation of both stiffness and strength where soil failure conditions and damage evolution functions are dealt 
separately. The failure condition curve (e.g., $S-N$ curve from cyclic triaxial or simple shear tests) can be expressed in two forms: the logarithm model [z839] or the semi-logarithm model [z940]. The damage evolution is evaluated by means of a stress-independent elliptical hardening/degradation function [2432], used to estimate both the stiffness and strength hardening/degradation factors:

$$
\delta_{\varsigma}=1+\left(\delta_{m \zeta}-1\right)\left[1-(1-D)^{\theta_{-}}\right]^{1 / \theta_{\varsigma}}
$$

where the subscript $\zeta$ stands for either $k$, the stiffness degradation factor, or s, the strength degradation factor. $\delta_{m \zeta}$ represents the minimum or maximum amount of degradation, $D$ the current cumulative damage and $\theta_{\zeta}$ the curve shape parameter. Figure 3a shows the hardening factor, $\theta_{\zeta}$, versus the number of cycles, $N$, the maximum hardening factor of $\delta_{m \zeta}(>1)$ can be achieved by either a concave $\left(\theta_{\zeta}<1\right)$ or a linear $\left(\theta_{\zeta}=1\right)$ or a convex $\left(\theta_{\zeta}>1\right)$ curve shape parameter. In Figure $3 \mathrm{~b}$ the degradation factor, $\theta_{\zeta}$, versus the number of cycles, $N$; the minimum degradation factor of $\delta_{m \zeta}(<1)$ can be attained by either a concave $\left(\theta_{\zeta}>1\right)$ or a linear $\left(\theta_{\zeta}=1\right)$ or a convex $\left(\theta_{\zeta}<1\right)$ curve shape parameter. The hardening/degradation factor remains constant as $\delta_{m \zeta}$ is reached. It is worth mentioning that the cyclic soil degradation and hardening is linked to an increase or decrease in the mean effective confining pressure due to the void ratio and pore pressure changes. The fatigue model used in this paper might be also used for simulating the effects of the liquefaction in terms of degrading behaviour (Allotey and El Naggar [23, 32] ) but neglects the lateral spreading that can be accounted for by adding further inertial loads to the pile (e.g. see Brandenberg et al., [41]). In this paper, non-liquefiable soil deposits are considered.

Radiation Damping

Radiation damping, caused by wave propagation away from the foundation, is modelled as a non-linear dashpot in parallel with a non-linear spring so that the damping is constant at each time step and is proportional to the current stiffness. The dashpot coefficient is based on the recommendations of Novak et al. [3042] and is used to represent the loss of energy due to outgoing stress waves that the pile transmits to the soil. With this formulation, when the system exhibits large displacements, the response 
is generally non-linear and the total damping is mainly due to hysteretic damping. The model also predicts small values of radiation damping when movement occurs in the slack zone and null radiation damping for the case of a pure gap. Therefore, a general reduction in radiation damping is expected for degrading systems, and an increase for hardening systems.

\section{PARAMETRIC INVESTIGATION}

A comprehensive parametric study is carried out to analyse the effects of soil non-linearity and soil degradation on the performance of floating and end-bearing single piles with fixed head condition.

Three different types of soil, characterized by suitable geotechnical parameters, are investigated in order to evaluate their nonlinear behaviour under seismic loading. The seismic input is defined at the seismic bedrock considering four different real accelerograms selected in order to represent different seismic hazard levels. Incremental Dynamic Analyses (IDAs) are performed to investigate the soil-pile interaction phenomena as the intensity of ground motion increases.

\section{Analysis cases and model description}

Six different homogeneous and four bi-layer soil deposits each one with three different soil types, dry sands (DS), saturated sands (SS) and saturated clay (SC), are considered, as shown in Figure 4a. For the homogeneous soil deposits two shear wave velocity values, $V_{s}=100 \mathrm{~m} / \mathrm{s}$ and $V_{s}=200 \mathrm{~m} / \mathrm{s}$ are considered. These are obtained by varying the soil type and properties, as presented in Table 1 . The two-layered soil deposit, which has an overall thickness of $30 \mathrm{~m}$, is characterized by the shear wave velocity of $\mathrm{V}_{\mathrm{s}}=100 \mathrm{~m} / \mathrm{s}$ for the top $15 \mathrm{~m}$ thick layer and $\mathrm{V}_{\mathrm{s}}=300 \mathrm{~m} / \mathrm{s}$ for the bottom $15 \mathrm{~m}$ thick layer. Table 2 shows the soil type and properties for the bi-layers cases.

In both the homogeneous and bi-layer cases, the deposit rests upon a uniform linear visco-elastic bedrock characterized by a shear wave velocity $\mathrm{V}_{\mathrm{s}}=800 \mathrm{~m} / \mathrm{s}$ and soil damping ratio $\xi=5 \%$. The foundation consists of a single vertical fixed-head pile with a circular cross section of diameter of $d=1.5$ $\mathrm{m}$ and a total length $L_{\mathrm{p}}=20 \mathrm{~m}$. The concrete used for the pile has a Young's modulus of $E_{p}=3 \times 10^{7}$ $\mathrm{kPa}$ and a density of $\rho_{p}=2.5 \mathrm{Mg} / \mathrm{m}^{3}$. The pile is modelled as a beam and is discretized into $0.5 \mathrm{~m}$ long 
beam elements to achieve a suitable level of accuracy (Figure 4b). Non-linear springs (spring-stiffness proportional dashpot combinations) are attached to each pile node in both sides (as shown in Figure 4c). The initial confining pressure is modelled by imposing a pre-straining displacement to the springs by considering a coefficient of lateral earth pressure $\mathrm{K}_{\mathrm{H}}$ equal to 1.0 in order to consider the effect of pile installation on the surrounding soil. The soil-pile interaction problem is investigated by creating a finite element model in SeismoStruct [3143] as depicted in Figure 5. Dynamic time-history ground motion accelerations are applied to each non-structural node connecting the pile to the soil link element in order to capture the effect of seismic wave propagation through the soil deposit.

\section{Soil-pile interaction model description and parameter estimation}

The Allotey and El Naggar [1923, 2432] degrading polygonal hysteretic link elements are used for simulating the soil-pile interaction. These elements are attached to both sides of the pile in order to account for the gap formation. To fully define the soil-pile interaction model, several parameters are calibrated from the soil data as reported in the following sections.

Backbone curves

The backbone curves used as reference in this work are the API-recommended p-y curves for sands and soft and stiff clay (API [3244]). The lateral soil resistance-pile deflection (p-y) relationship for sand for a specific depth $z$, is approximated asfollows:

$$
p=0.9 \cdot p_{u} \cdot \tanh \left(\frac{k \cdot z}{0.9 \cdot p u} y\right)
$$

where $k$ is the soil initial modulus of subgrade reaction and $p_{u}$ is the ultimate bearing capacity determined as the smallest value between the shallow and deep ultimate resistance, $p_{u s}$ and $p_{u d}$, respectively, determined as follows :

$$
\begin{gathered}
p_{u s}=\left(C_{1} \cdot H z+C_{2} \cdot D d\right) \cdot \gamma \cdot z \\
p_{u d}=\left(C_{3} \cdot D d\right) \cdot \gamma \cdot z
\end{gathered}
$$

where $\gamma$ is the effective soil unit weight, $\rightarrow d$ is the pile diameter and $C_{1}, C_{2}$, and $C_{3}$ are coefficients as a function of the frictional angle or shear strength angle, $\varphi$ and are determined by the following equations: 


$$
\begin{aligned}
& \left.\left.C_{1}=\tan (\beta) \cdot\left[(\tan \beta)^{2} \cdot \tan {\underset{2}{(})}_{\frac{\varphi}{2}}+K_{H}\left[\tan (\varphi) \cdot \sin \beta \cdot \underset{\cos \left(\frac{\varphi}{2}\right)}{\left(\frac{1}{2}\right.}+1\right)\right]-\tan \frac{(}{2}\right)\right]_{2} \\
& C=(\tan \beta)^{2}-\left[\tan \left(-{ }^{\varphi}+{ }^{\pi}\right)^{2}\right. \\
& \left.2 \quad-\begin{array}{cc}
- \\
2 & 2
\end{array}\right] \\
& C=(\tan \beta)^{4} \cdot\left[(\tan \beta)^{2}+K(\tan \varphi)\right]-\left[\tan \left(-{ }^{\varphi}+{ }^{\pi}\right)^{2}\right. \\
& 3 \\
& H
\end{aligned}
$$

in which $\beta=\frac{\varphi}{2}+\frac{\pi}{2}$.

On the other hand, the lateral soil resistance-pile deflection relationships for soft and stiff clays are determined by tabular curves defined in [3244] and reported in Figure 6; the ultimate resistance $p_{u}$ is determined by the following criteria:

$$
\begin{array}{cc}
p_{u s}=3 G c_{u}+\gamma \cdot z z+J \cdot \frac{C_{u} \cdot z z}{\underline{D} d} & \text { for } H z<\frac{6 \underline{\underline{D} d}}{\frac{\gamma \cdot D d}{c \bar{\epsilon} u}+J} \\
p_{u d}=9 \underline{C} c_{u} & \text { for } H z>\frac{6 \underline{D} d}{\frac{\gamma \cdot D}{c \epsilon_{u}}+J}
\end{array}
$$

where $J$ dimensionless empirical constant assumed as $J=0.5$ for soft clays and $J=1.5$ for stiff clays and $c_{u}$ is the undrained shear strength.

The geotechnical characteristics of the soils needed for defining the p-y curves used in this paper for the parametric investigation have been derived from the literature. Different levels of initial void ratio are defined consistently to achieve the dynamic characteristics of the cases analysed, both for homogeneous and bi-layer cases. For the dry sandy soil, a well graded Al (AASHTO Classification System) white silica sand is considered with particle sizes in the range of $0.075-0.59 \mathrm{~mm}, \mathrm{D}_{50}=0.288 \mathrm{~mm}, e_{\min }=$ 0.637 and $e_{\max }=1.046$ [3345]. Three levels of initial void ratio, $e_{0}=0.9$ (relative density, $\mathrm{Dr}=35 \%$, loose dry sand), $e_{0}=0.82$ ( $\mathrm{Dr}=55 \%$, medium dense dry sand), and $e_{0}=0.76(\mathrm{Dr}=70 \%$, dense dry sand) are considered. For the saturated cases, a fine uniformly graded Nevada sand [3446] with Coefficient of Uniformity, $\mathrm{Cu}_{-} C_{u_{-}}=1.5$ and $\mathrm{D}_{50}=0.15 \mathrm{~mm}$ has been considered. As in the dry case, different levels of void ratio are defined: $e=0.73$ ( $\mathrm{Dr}=42 \%$, loose saturated sand), $e=0.65(\mathrm{Dr}=$ $63 \%$, medium dense saturated sand) and $e=0.62$ ( $\mathrm{Dr}=71 \%$, dense saturated sand). For the saturated 
clayey cases, a normal consolidated Drammen clay [3547], a marine clay with a plasticity index PI = , a clay content of $45-55 \%$ and Liquid Limit, LL of 55 is considered. As done for the sand, three soil consistencies, i.e. soft, medium and stiff soil, are defined. Tables 1 and 2 exhaustively-summarizes the and mechanical values considered in the whole set of analyses performed, for homogeneous and bilayer cases respectively. Figure 6 shows the original API curves, assembled by using Eqs. 3-10 according to the soil properties reported in Table 1 and Table 2, and the four-segment curves fitting the API curves, which are used in the numerical model. The fitting parameters of the normalised backbone curves are also listed in Figure 6 where $f\left(K_{0}\right)$ and $g\left(K_{0}\right)$ indicate the stiffness values of the second and third branch, respectively, defined as a function of the initial dynamic stiffness $K_{0}=1.2 \cdot E_{s}$, where $E_{s}$ is the Young's soil modulus [3648]; it is worth noting that the shape of the backbone for sand is not dependent upon the ultimate pressure $p_{u}$, hence constant values are obtained.

Cyclic response and cyclic hardening/degradation

Table 2 shows the combination of different parameters required by the cyclic p-y model used for each soil type. Gapping is assumed to occur within the top third of the pile where the lateral confining pressure is lower. However, in dry sand, any developed gap will be simultaneously filled with falling soil (cave-in soil) and no permanent gap will be developed. The soil cave-in parameters are assumed to vary linearly with depth and to increase with the lateral confining pressure. Stiffness and strength degradation parameters are based on physical quantities deduced from the literature by fitting the data with the degradation model depicted in Figure 3; centrifuge tests are used for saturated sand [3749] and undrained cyclic triaxial compression tests for clay [29 $\underline{20}$ ] and finally, for dry sand a typical hardening response is considered [3850]. Model parameters of the BNWF depicted in Figure 5 are reported in 3 and 4.

\section{Definition of ground motion records and free-field displacements}

Under cyclic or dynamic loads, the soil exhibits hardening/degrading behaviour and can considerably change its strength and stiffness in response to applied stresses. The response of foundation then becomes strongly affected by the number of effective cycles of seismic loading and the Ground Motion 
Duration (GMD) could become an important parameter significantly affecting the piles response. The ground motion is usually characterised in terms of peak or integral parameters. Cosenza and Manfredi [3951] proposed a damage factor, $I_{D}$, that is related to the number of plastic cycles and, therefore, to the energy content of the earthquake, i.e.

$$
I_{D}=\frac{2 g \quad I_{A}}{\pi P G A P G V}
$$

where $I_{A}$ is the Arias Intensity and $P G A$ and $P G V$ are the peak ground acceleration and the peak ground velocity, respectively. In this study, 4 real ground motion records, defined at the outcropping bedrock, are selected from the Pacific Earthquake Engineering Research Center (PEER) database, to be representative of different duration scenarios: 'small duration' $\left(\mathrm{I}_{\mathrm{D}}<5\right)$ ), 'moderate duration' (ID $<16$ ) and 'large duration' $\left(I_{D}>22\right)$. Figure 7 shows the seismic acceleration time histories of the selected records with their values of damage factor $I_{D}$, duration, $t_{D}$, and effective record duration , $t_{E}$.

To investigate the effects of the GMD on the non-linear seismic response of the soil-pile system, each record is scaled to 4 progressively increasing levels of intensity using an iterative procedure. This procedure involves: firstly, applying a scale factor to the selected outcropping motion; secondly, performing a 1D linear-equivalent site response analysis; lastly, adjusting the input motion iteratively until the spectral acceleration of the surface motion converges to the desired value. In this work, the intensity measures (IMs) are selected to correspond to the following values of spectral surface acceleration $0.1 \mathrm{~g}, 0.2 \mathrm{~g}, 0.4 \mathrm{~g}$, and $0.6 \mathrm{~g}$, corresponding to the fundamental period of $0.8 \mathrm{~s}$. The employed iterative procedure generates surface input motions with the same IM for each soil profile without neglecting the local site effects on the frequency content of the signal; this distinguishes from a conventional deconvolution analysis where the surface input motion is given. Moreover, it is a further improvement with respect to selecting the same ground motion for every soil profiles as done in Gerolymos et al. [4052]. It is worth noting that the proposed procedure aims to generate site-specific 
earthquake input motions characterized by the same intensity at the ground surface in order to facilitate the comprehension of the effects of the near-field nonlinearities on the performance of the pile.

Therefore, $1 \mathrm{D}$ site response analyses are performed considering different degradation and damping ratio curves for clayey and sandy soils [4153-4456] (Figure 8a-b). A total number of 16 ground motions, defined at the outcropping bedrock, are obtained for each soil profile. In the $2^{\text {nd }}$ step of the analysis, the calculated motion at each elevation is applied as input motion to the soil spring along the pile length, and the pile response is evaluated.

\section{RESULTS}

The results obtained from the site response analysis (for the definition of the free-field displacements) and the nonlinear soil-pile interaction are reported. The influences of the ground motion intensity and of the GMD on the kinematic soil-pile interaction for the different soil types are discussed.

\section{Site response analysis for the free-field displacements}

The 1D linear equivalent site response analyses are adopted to capture the local site effects (e.g. amplifications) and to evaluate the free-field displacement within each soil profile, starting from the 16 ground motion records previously defined. Suitable shear modulus degradation and damping ratio curves, as illustrated in Figure 8, are used in the analysis to account for soil non-linearity. The iterative procedure is performed in order to achieve the IM of $0.1 \mathrm{~g}, 0.2 \mathrm{~g}, 0.4 \mathrm{~g}$, and $0.6 \mathrm{~g}$ corresponding to the fundamental soil deposit period of $0.8 \mathrm{~s}$.

Figure 9 shows the acceleration elastic response spectra at the ground surface motion for the San Fernando earthquake calculated by the site response analysis for the soil profiles considered: dry sand soil; saturated sand; and saturated clay. The points on the curves indicated by circles demonstrate the convergence between the achieved spectral acceleration value and the IMs previously defined. 
Saturated and dry sands, with same shear wave velocity Vs (100DS/100SS, 100300DS/100300SS or 200DS/200SS), exhibit mostly the same acceleration response spectrum at the ground surface, owing to similar cyclic nonlinear behaviour. Moreover, a significant-shift in the site's fundamental periods is observed as the intensity level increases, especially in the bi-layer deposit. This behaviour is less evident in saturated clays because of the larger linear cyclic shear strain threshold of clays compared to the sands, as shown in Figure 8. Similar results are also obtained for the other ground motion records.

In Figure 10, the Arias intensity of the surface ground acceleration is plotted against the increment of the intensity measure for every investigated earthquake event and soil type. The results show that the Arias intensities of the surface ground motions obtained by the proposed procedure are similar except for the Chi-Chi earthquake event in soft soils where its value appears consistently higher than the other cases. It is worth noting that both spectral acceleration and Arias intensity can be used as good candidate IMs to evaluate the kinematic interaction in nonlinear soil, as observed in Bradley et al. [57] and both will be used to evaluate the seismic performance of the single pile.

\section{Influence of intensity and duration of ground motion}

Nonlinear kinematic interaction analyses are performed by accounting for soil nonlinearities such as soil yielding, cyclic degradation of soil stiffness and strength, soil-pile gap formation with or without cave-in and recompression, caused by large relative soil-pile lateral displacements. The free-field displacements achieved from the $1^{\text {st }}$ step are applied to the lateral nonlinear springs attached to the shaft. Results are reported in terms of envelops of bending moment within the pile.

The graphs in Figure 10 11 show the envelops of maximum and minimum bending moments within the pile obtained from the IDAs for the soil profiles with shear wave velocity, $\mathrm{V}_{\mathrm{s}}=100 \mathrm{~m} / \mathrm{s}$ (i.e., 100DS, 100SS and 100SC) for the 4 selected records. It is evident that the bending moments related to saturated and dry sands (100SS and 100DS) are comparable, while the ones relevant to the saturated clays (100SC) are almost an order of magnitude smaller, although the IMs achieved at the ground surface, are the same for the three soil types. Differences between sandy and clayey deposits are principally due to 
their different nonlinear behaviour arisen from the site response analyses. On the other hand, since the results in dry and saturated sandy deposits are similar in the case of earthquakes with small duration, such as for the events of San Fernando Earthquake and Loma Prieta Earthquake, the cyclic behaviour and the formation of gapping do not significantly affect the overall pile behaviour; accordingly, an equivalent linear analysis can be performed. Conversely, for earthquakes with large duration, the effect of the cyclic degradation/hardening and the gapping affect the seismic response significantly, resulting in a marked difference between the bending moments derived in dry sandy cases from those obtained in saturated sandy profiles; nonlinear analysis is thus required. The bending moment distributions are generally characterized by two relative maximum values, a peak value at the pile head due to the fixed head condition and the second one occurring at a certain depth along the pile related to the free field soil deformation of the deposit. Moreover, for the 100DS profile, at lower levels of intensity, the maximum bending moment is localized along the pile at a depth of about $2 / 3 \mathrm{~L}_{\mathrm{p}}$ below the pile head for all the selected records and the bending moment at the head is generally much smaller. With increasing levels of seismic intensity, the maximum bending moment, at about $2 / 3 \mathrm{~L}_{\mathrm{p}}$ below the pile head decreases and the bending moment distribution becomes more severe in the upper part of the pile. Similar observations are made for the 100SS profile where at high levels of intensity, the maximum bending moment is attained at the pile head. Remarkably, the bending moments decrease along the pile with a higher rate in saturated sand than what occur for the dry sandy cases because of the cyclic degradation effect. The loss of the lateral bearing along the shaft causes the increase of the stresses at the pile head where the structural rotational restraint is applied. Finally, the 100SC bending moment profiles have similar shape of the saturated sandy cases achieving the maximum value at the head of the pile while gradually decrease along the pile although they are always characterized by smaller values of bending moment.

Figure $11-12$ depicts the results obtained for the homogeneous profiles, which are characterized by $\mathrm{V}_{\mathrm{s}}$ $=200 \mathrm{~m} / \mathrm{s}$; the distribution of the bending moment along the pile is similar to those obtained at low intensity for the previous cases with softer soils. Type of soil and ground motion duration does not 
significantly affect the bending moment profile; accordingly, equivalent linear analysis provides reliable results for medium-stiff soildeposit.

Figure $12 \underline{13}$ shows the envelops of maximum and minimum bending moments within the pile obtained from the IDAs for the bi-layer soil profiles with shear wave velocity equal to $100 \mathrm{~m} / \mathrm{s}$ for the upper layer and equal to $300 \mathrm{~m} / \mathrm{s}$ for the bottom layer (i.e., 100300DS, 100300SS and 100300SC) for the 4 selected records. The distribution of the bending moment presents two relative maximum values that occur at the pile head and at the interface between the two layers. As previously described, the bending moments within the pile in the saturated clay are appreciably lower than those obtained for the sandy cases. For the lowest level of intensity (0.1g), the maximum bending moment usually occurs at the interface between the layers. This bending moment distribution is also observed in dry sand cases (100300DS) for high intensity levels while in saturated soils (i.e. 100300SS and 100300SC cases), the bending moment at the interface decreases with the increase of the level of intensity. On the other side, it increases at the head level until reaching the maximum value. The decrease of the moment at the interface is caused by the reduction of the layer stiffness contrast because of the soil yielding. Moreover, long ground motion duration increases the nonlinear effects on the seismic response of the pile on saturated sands such as the formation of gap and the cyclic degradation that produces soil strength and stiffness decrease.

In conclusion, with increasing seismic intensity, saturated soils exhibit significant non-linear behaviour (cyclic degradation of soil stiffness and strength, soil-pile gap formation with or without cave-in and recompression, soil yielding) and nonlinear analysis is required to obtain reliable results. In dry sandy soils, if the ground motion intensity is not high, an equivalent linear analysis can be carried out.

These observations are summarized in the-Figure 14 where the increment of the maximum bending is plotted against the intensity measure. By comparing with the Arias intensity curves reported in Figure 10, it can be observed that the soil nonlinearities affect the kinematic response by decreasing the 
maximum response with the increase of the duration of the earthquake event, especially in saturated soils where a nonlinear softening behaviour is exhibited.

Finally, the foundation input motion is analysed in Figure 15 in terms of maximum relative displacement of the pile head as opposed to the free-field motion. The foundation input displacement at the head of the pile is larger in saturated than in dry soils due to the formation of the softening hysteresis soil-pile curves as reported in Figure 1a for saturated sands and Figure 1d for saturated soft clays. The nonlinear effects are seen exacerbated for long ground motion duration earthquakes since the differences of the relative displacements between dry and saturated soil, caused by the large duration motions such as ChiChi and Imperial Valley events are higher than the ones caused by small duration motion such as San Fernando and Loma Prieta events.

Finally Therefore, it may be concluded that the GMD affects the nonlinear seismic response of the pile, especially in saturated soil. In cases characterised by large duration scenarios, nonlinearities affect the pile behaviour even for low intensity level of $0.2 \mathrm{~g}$.

\section{COMPARISON WITH LINEAR ANALYSIS}

The obtained results are compared with those derived from a linear soil-pile interaction model [45 $\underline{33}$ ]. Linear kinematic interaction analyses are performed applying a two-step uncoupled procedure. In the $1^{\text {st }}$ step, the free-field displacements within the deposit along the pile are defined by means of the same linear-equivalent site response procedures applied for the previous analysis. In the $2^{\text {nd }}$ step, the soil-pile interaction is evaluated in the frequency domain using the linear soil-pile interaction model proposed by Dezi et al. [4533]. In this method, the analyses assumed linear behaviour for the pile and the soil. The pile is modelled as a beam element embedded in the soil represented by independent horizontal infinite layers and no soil-pile gap is allowed to occur during the motion. Figure $13-16$ shows the envelops of maximum and minimum bending moments along the pile shaft for all soil profiles considered for the Imperial Valley earthquake classified as large duration scenario and scaled to the medium-high IM equal to $0.6 \mathrm{~g}$. 
The results obtained from the linear model are in good agreement with those obtained with the nonlinear model except when the soil is a soft saturated sand in which the bending moment within the pile is over predicted if a linear soil model is considered. This discrepancy is due the nonlinear behaviour of the soil material in addition to the relevant cyclic degradation effects occurring in saturated sand.

Finally, iIn Figure 1417, the same analysis is proposed by considering a low IM equal to $0.1 \mathrm{~g}$. It is worth noting that a good matching to the linear model is obtained confirming the considerations drawn from the previous section.

\section{GONGLUSIONSCONCLUDINGREMARKS}

Incremental Dynamic Analyses have been performed to evaluate the effects of GMD and soil nonlinearity on the kinematic interaction of single fixed-head piles in homogeneous and bilayer soil profiles such as dry sand, saturated sand and saturated clay. A two-step uncoupled procedure has been followed in the analysis: firstly, the free field motion is evaluated considering an equivalent linear site response analysis; secondly, the stress resultants in the pile were evaluated using the Allotey and El Naggar [1923, 2432] BNWF model, which is able to account for cyclic soil degradation/hardening, soil and structural yielding, slack zone development and radiation damping. The model parameters have been calibrated from soil data obtained from the literature. The following conclusions may be drawn:

at high levels of intensity, the maximum bending moment is attained at the pile head while it decreases below the pile head in the lower part of the pile;

the bending moments decrease along the pile with a higher rate in saturated sand than what occur for the dry sandy cases because of the cyclic degradation effect;

the effect of the cyclic degradation/hardening and the gapping is exacerbated with large duration earthquakes;

in dry sandy soils or for earthquake with a low-medium intensity measure, a linear soil-pile interaction model can be adopted. 
It is worth mentioning that the BNWF approach can be used along with linear or equivalent linear site response analysis but for strong ground motions, the pile response can be predicted more accurately within the framework of fully nonlinear direct analysis where both near- and far-field are coupled. In this case, more complex approaches using 3D finite element methods should be used. 


\section{REFERENCES}

[1] E. Kausel, "Early history of soil-structure interaction," Soil Dynamics and Earthquake Engineering, vol. 30, no. 9, pp. 822-832, Sep. 2010.

[2] E. Kausel and J. Roësset, "Soil amplification: some refinements," International Journal of Soil Dynamics and Earthquake Engineering, vol. 3, no. 3, pp. 116-123, Jul. 1984.

[3] E. Kausel, R. V. Whitman, J. P. Morray, and F. Elsabee, “The spring method for embedded foundations,” Nuclear Engineering and Design, vol. 48, no. 2-3, pp. 377-392, Aug. 1978. [4] N. W. Trombetta, H. B. Mason, Z. Chen, T. C. Hutchinson, J. D. Bray, and B. L. Kutter, "Nonlinear dynamic foundation and frame structure response observed in geotechnical centrifuge experiments," Soil Dynamics and Earthquake Engineering, vol. 50, pp. 117-133, Jul. 2013.

[5] R. W. Boulanger, C. J. Curras, B. L. Kutter, D. W. Wilson, and A. Abghari, "Seismic Soil-PileStructure Interaction Experiments and Analyses," Journal of Geotechnical and Geoenvironmental Engineering, vol. 125, no. 9, pp. 750-759, Sep. 1999S. Dashti, J. D. Bray, J. M. Pestana, M. Riemer, and D. Wilson, "Centrifuge Testing to Evaluate and Mitigate Liquefaction-InducedBuilding Settlement Mechanisms," Journal of Geotechnical and Geoenvironmental Engineering, vol. 136, no. 7, pp. 918-929, Jul. 2010.

[6] D. Pitilakis, M. Dietz, D. M. Wood, D. Clouteau, and A. Modaressi, "Numerical simulation of dynamic soil-structure interaction in shaking table testing," Soil Dynamics and Earthquake Engineering, vol. 28, no. 6, pp. 453-467, Jun. 2008.

[7] M. R. Massimino and M. Maugeri, "Physical modelling of shaking table tests on dynamic soilfoundation interaction and numerical and analytical simulation," Soil Dynamics and Earthquake Engineering, vol. 49, pp. 1-18, Jun. 2013.

[8] G. Biondi, M. R. Massimino, and M. Maugeri, "Experimental study in the shaking table of the input motion characteristics in the dynamic SSI of a SDOF model," Bulletin of Earthquake Engineering, vol. 13, no. 6, pp. 1835-1869, Jun. 2015.

[9] G. Abate and M.R. Massimino. Dynamic soil-structure interaction by experimental and numerical modelling. Rivista Italiana di Geotecnica. Vol. 50, no 2, pp. 44-70. 2016. [810] P. J. Meymand, "Shaking table scale model tests of nonlinear soil-pile-superstructure interaction in soft clay," PhD Thesis, University of California, Berkeley, 1998.

[911] A. Chidichimo, R. Cairo, G. Dente, C. A. Taylor, and G. Mylonakis, "1-g Experimental investigation of bi-layer soil response and kinematic pile bending," Soil Dynamics and Earthquake Engineering, vol. 67, pp. 219-232, Dec. 2014.F. Moccia, "Seismic Soil Pile Interaction Experimental Evidence," PhD Thesis, Universita’ Degli Studi Di Napoli Federico II, 2009. [1012] M. G. Durante, L. Di Sarno, G. Mylonakis, C. A. Taylor, and A. L. Simonelli, "Soil-pilestructure interaction: experimental outcomes from shaking table tests: Shake Table Studies of SoilPile-Structure Interaction,” Earthquake Engineering \& Structural Dynamics, vol. 45, no. 7, pp. 1041-1061, Jun. 2016.

[11스] B. K. Maheshwari, K. Z. Truman, M. H. E. Naggar, and P. L. Gould, “Three-dimensional finite element nonlinear dynamic analysis of pile groups for lateral transient and seismic excitations," Canadian Geotechnical Journal, vol. 41, no. 1, pp. 118-133, Feb. 2004.

[14] B. K. Maheshwari, K. Z. Truman, M. H. El Naggar, and P. L. Gould, "Three-dimensional nonlinear analysis for seismic soil-pile-structure interaction," Soil Dynamics and Earthquake Engineering, vol. 24, no. 4, pp. 343-356, Jun. 2004.

[15] B. K. Maheshwari, K. Z. Truman, P. L. Gould, and M. H. El Naggar, "Three-Dimensional Nonlinear Seismic Analysis of Single Piles Using Finite Element Model: Effects of Plasticity of Soil," International Journal of Geomechanics, vol. 5, no. 1, pp. 35-44, Mar. 2005.

[16] F. Grassi and M. R. Massimino, "Evaluation of kinematic bending moments in a pile foundation using the finite element approach," WIT Transactions on the Built Environment, Vol. 104, 2009, pp. 479-488,2016. 
[1217] C. Luo, X. Yang, C. Zhan, X. Jin, and Z. Ding, "Nonlinear 3D finite element analysis of soil-pile-structure interaction system subjected to horizontal earthquake excitation,” Soil Dynamics and Earthquake Engineering, vol. 84, pp. 145-156, May 2016.[13] R. W. Boulanger, C. J. Curras, B. L. Kutter, D. W. Wilson, and A. Abghari, "Seismic Soil-Pile-Structure Interaction Experiments and Analyses," Journal of Geotechnical and Geoenvironmental Engineering, vol. 125, no. 9, pp. 750-759, Sep. 1999.

[1418] M. H. El Naggar and K. J. Bentley, "Dynamic analysis for laterally loaded piles and dynamic p-y curves,” Canadian Geotechnical Journal, vol. 37, no. 6, pp. 1166-1183, Dec. 2000. [1519] Brown, D.A., O’Neill, M.W., Hoit, M., McVay, M., El Naggar,M.H., and Chakrabotry, S. 2001. Static and dynamic lateralloading of pile groups. NCHRP Report 461, Transportation Research Board, Washington, D.C

[1620] N. Gerolymos and G. Gazetas, "Constitutive Model For 1-D Cyclic Soil Behaviour Applied To Seismic Analysis Of Layered Deposits,” SOILS AND FOUNDATIONS, vol. 45, no. 3, pp. 147159, 2005.

[1721] N. Gerolymos and G. Gazetas, "Phenomenological Model Applied To Inelastic Response Of Soil-Pile Interaction Systems,” SOILS AND FOUNDATIONS, vol. 45, no. 4, pp. 119-132, 2005.

[1822] N. Allotey, "Non-linear soil-structure interaction in performance-based design," University of Western Ontario, London, Ontario, 2006.

[1923] N. Allotey and M. H. El Naggar, "Generalized dynamic Winkler model for nonlinear soilstructure interaction analysis,” Canadian Geotechnical Journal, vol. 45, no. 4, pp. 560-573, Apr. 2008.

[24] N. Allotey and M. H. El Naggar, "A numerical study into lateral cyclic nonlinear soil-pile response," Canadian Geotechnical Journal, vol. 45, no. 9, pp. 1268-1281, Sep. 2008.

[25] M. Heidari, M. Jahanandish, H. El Naggar, and A. Ghahramani, "Nonlinear cyclic behavior of laterally loaded pile in cohesive soil," Canadian Geotechnical Journal, vol. 51, no. 2, pp. 129-143, Feb. 2014

[Z026] M. Heidari, H. El Naggar, M. Jahanandish, and A. Ghahramani, "Generalized cyclic p-y curve modeling for analysis of laterally loaded piles,” Soil Dynamics and Earthquake Engineering, vol. 63, pp. 138-149, Aug. 2014.

[2127] N. A. Alexander, "Estimating the nonlinear resonant frequency of a single pile in nonlinear soil,” Journal of Sound and Vibration, vol. 329, no. 8, pp. 1137-1153, Apr. 2010.

[28] K. J. Bentley and M. H. E. Naggar, "Numerical analysis of kinematic response of single piles," Canadian Geotechnical Journal, vol. 37, no. 6, pp. 1368-1382, Dec. 2000.

[2229] M. Raghunandan and A. B. Liel, "Effect of ground motion duration on earthquake-induced structural collapse,” Structural Safety, vol. 41, pp. 119-133, Mar. 2013.

[2330] I. Iervolino, G. Manfredi, and E. Cosenza, "Ground motion duration effects on nonlinear seismic response,” Earthquake Engineering \& Structural Dynamics, vol. 35, no. 1, pp. 21-38, Jan. 2006.

[31] D. Vamvatsikos and C. A. Cornell, "Incremental dynamic analysis," Earthquake Engineering \& Structural Dynamics, vol. 31, no. 3, pp. 491-514, Mar. 2002.

[2432] N. Allotey and M. H. El Naggar, "A Consistent Soil Fatigue Framework Based on the Number of Equivalent Cycles,” Geotechnical and Geological Engineering, vol. 26, no. 1, pp. 6577, Aug. 2007.

[33] F. Dezi, S. Carbonari, and G. Leoni, "A model for the 3D kinematic interaction analysis of pile groups in layered soils,” Earthquake Engineering \& Structural Dynamics, vol. 38, no. 11, pp.

1281-1305, Sep. 2009.

[34] F. Dezi, S. Carbonari, and G. Leoni, "Static equivalent method for the kinematic interaction analysis of single piles," Soil Dynamics and Earthquake Engineering, vol. 30, no. 8, pp. 679-690, Aug. 2010. 
[35] F. Dezi, S. Carbonari, and M. Morici, "A numerical model for the dynamic analysis of inclined pile groups: A Numerical Model for the Dynamic Analysis of Inclined Pile Groups,” Earthquake Engineering \& Structural Dynamics, vol. 45, no. 1, pp. 45-68, Jan. 2016.

[2536] R. M. Pyke, “Nonlinear Soil Models for Irregular Cyclic Loadings,” Journal of the Geotechnical Engineering Division, ASCE, vol. 105, no. 6, pp. 715-726, 1979.

[2637] K. Yasuhara, S. Murakami, B.-W. Song, S. Yokokawa, and A. F. L. Hyde, "Postcyclic Degradation of Strength and Stiffness for Low Plasticity Silt,” Journal of Geotechnical and Geoenvironmental Engineering, vol. 129, no. 8, pp. 756-769, Aug. 2003.

[2738] K. Yasuhara, "Postcyclic undrained strength for Cohesive Soils,” Journal of Geotechnical Engineering, vol. 120, no. 11, pp. 1961-1979, Nov. 1994.

[2839] S. S. Sharma and M. Fahey, "Evaluation of Cyclic Shear Strength of Two Cemented Calcareous Soils,” Journal of Geotechnical and Geoenvironmental Engineering, vol. 129, no. 7, pp. 608-618, Jul. 2003.

[2940] M. Hyodo, Y. Yamamoto, and M. Sugiyama, "Undrained cyclic shear behavior of normally consolidated clay subjected to initial static shear stress," Soils and Foundations, vol. 34, no. 4, pp. 111, 1994. M. Hyodo, H. Murata, N. Yasufukt, and T. Fuijii, "Undrained cyclic shear strength and residual shear strain of saturated sand by cyclic triaxial tests," Soils and Foundations, vol. 31, no. 3, pp. 60-76, 1991.

[41] S. J. Brandenberg, R. W. Boulanger, B. L. Kutter, and D. Chang, "Behavior of Pile

Foundations in Laterally Spreading Ground during Centrifuge Tests," Journal of Geotechnical and Geoenvironmental Engineering, vol. 131, no. 11, pp. 1378-1391, Nov. 2005.

[3042] M. Novak, T. Nogami, and F. Aboul-Ella, "Dynamic Soil Reactions for Plane Strain Case," Journal of the Engineering. Mechanics Division, ASCE, vol. 104, pp. 953-959, 1978.

[31느] Seismosoft, SeismoStruct. Seismosoft, 2013.

[3244] API, "Recommended practice for planning, designing and constructing fixed offshore platforms,” American Petroleum Institute (API), Washington, D.C, 2007.

[3345] M. H. T. Rayhani and M. H. Naggar, "Dynamic Properties of Soft Clay and Loose Sand from Seismic Centrifuge Tests,” Geotechnical and Geological Engineering, vol. 26, no. 5, pp. 593602, Jun. 2008.

[3446] D. W. Wilson, “Soil-pile-superstructure interaction in liquefying sand and soft clay," PhD Thesis, University of California, Davis, Department of Civil and Environmental Engineering, 1998. [3547] K. H. Andersen, A. Kleven, and D. Heien, "Cyclic Soil Data for Design of Gravity Structures,” Journal of Geotechnical Engineering, vol. 114, no. 5, pp. 517-539, May 1988. [3648] D. Badoni and N. Makris, "Nonlinear response of single piles under lateral inertial and seismic loads," Soil Dynamics and Earthquake Engineering, vol. 15, no. 1, pp. 29-43, Jan. 1996.

[3749] R. Popescu and J. H. Prevost, "Centrifuge validation of a numerical model for dynamic soil liquefaction,” Soil Dynamics and Earthquake Engineering, vol. 12, no. 2, pp. 73-90, Jan. 1993. [3850] D. C. Lo Presti, A. Cavallaro, M. Maugeri, O. Pallara, and F. Ionescu, "Modeling of hardening and degradation behaviour of clay and sands during cyclic loading," in 12WCEE, Auckland, New Zealand, 2000.

[3951] E. Cosenza and G. Manfredi, "The improvement of the seismic-resistant design for existing and new structures using damage criteria," in Seismic design methodologies for the next generation of codes, P. Fajfar and H. Krawinkler, Eds. Balkema, Rotterdam, the Netherlands, 1997, pp. 119130.

[4052] N. Gerolymos, V. Drosos, and G. Gazetas, "Seismic response of single-column bent on pile: evidence of beneficial role of pile and soil inelasticity,” Bulletin of Earthquake Engineering, vol. 7, no. 2, pp. 547-573, Apr. 2009.

[4153] I. M. Idriss, "Response of Soft Soil Sites during Earthquakes," in Memorial Symposium to honor Professor Harry Bolton Seed, Berkeley, California, 1990, vol. II.

[4254] L. Reese, W. Cox, and F. Koop, "Field Testing and Analysis of Laterally Loaded Piles om Stiff Clay,” 1975. 
[4355] H. B. Seed and I. M. Idriss, "Influence of soil conditions on ground motions during earthquakes,” Journal of Soil Mechanics and Foundations Division, ASCE, vol. 95, no. 1, pp. 99138, 1969.

[4456] M. Vucetic and R. Dobry, “Effect of Soil Plasticity on Cyclic Response,” Journal of Geotechnical Engineering, vol. 117, no. 1, pp. 89-107, Jan. 1991.

[57] B. A. Bradley, M. Cubrinovski, and R. P. Dhakal, "Performance-Based Seismic Response of Pile Foundations," 2008, pp. 1-11.

[45] F. Dezi, S. Garbonari, and G. Leoni, "A model for the 3D kinematic interaction analysis of pile groups in layered soils," Earthquake Engineering \& Structural Dynamics, vol. 38, no. 11, pp. 1281-1305, Sep. 2009.

LIST OF SYMBOLSNOMENCLATURE

\begin{tabular}{|l|l|}
\hline$c_{u}$ & undrained shear strength \\
\hline$C_{u}$ & coefficient of uniformity \\
\hline$\underline{\mathrm{d}}$ & pile diameter \\
\hline $\mathrm{D}$ & current cumulative damage \\
\hline $\mathrm{D}_{\mathrm{r}}$ & relative density \\
\hline $\mathrm{D}_{0}$ & diameter of the soil particle for which $50 \%$ of the particles are finer \\
\hline$e$ & Void ratio \\
\hline$e_{0}$ & Initial void ratio \\
\hline$e_{\max }$ & Maximum void ratio \\
\hline$e_{\min }$ & Minimum void ratio \\
\hline$E_{p}$ & elastic modulus of the pile \\
\hline$E_{p s}$ & $\underline{\text { elastic modulus of the pilesoil }}$ \\
\hline$\underline{I_{A}}$ & $\underline{\text { Arias Intensity }}$ \\
\hline$\underline{I_{D}}$ & $\underline{\text { damage factor }}$ \\
\hline$y$ & $\underline{\text { horizontal coordinate }}$ \\
\hline$L_{p}$ & pile length \\
\hline$p$ & lateral dynamic load \\
\hline$p_{u}$ & ultimate lateral force \\
\hline
\end{tabular}




\begin{tabular}{|c|c|}
\hline pur & force at the onset of unloading or reloading \\
\hline$p_{u s}$ & shallow ultimate bearing capacity \\
\hline$p_{u d}$ & deep ultimate bearing capacity \\
\hline$\underline{\underline{\kappa}}$ & Pike’s scale factor \\
\hline$k$ & Lateral-lateral nonlinear soil-pile reaction modulus \\
\hline$\underline{\underline{K_{0}}}$ & backbone curve initial stiffness \\
\hline$\overline{\mathrm{K}_{\mathrm{H}}}$ & coefficient of lateral earth pressure \\
\hline $\begin{array}{l}t \\
-\end{array}$ & time \\
\hline$\underline{t_{D}}$ & Total duration of the recorded signal \\
\hline$\underline{t_{E}}$ & duration of the strong shaking phase \\
\hline$\underline{\mathrm{V}_{\mathrm{s}}}$ & shear wave velocity \\
\hline $\mathrm{z}$ & vertical coordinates for the pile depth \\
\hline$\beta$ & Rankine’s active shear plane failure angle \\
\hline$\gamma$ & effective soil unit weight \\
\hline$\underline{\mu_{p}}$ & Mass per unit length of the pile \\
\hline$\psi$ & effective soil unit weight \\
\hline$\delta_{m \kappa}, \delta_{m s}$ & stiffness and strength hardening/degradation factors, respectively \\
\hline $\begin{array}{l}\delta_{k}, \\
\delta_{s} \oint_{m}\end{array}$ & $\begin{array}{l}\text { stiffness and strength degradation parameters, respectivelystrength } \\
\text { hardening/degradation factors }\end{array}$ \\
\hline$\oint_{s}$ & strength degradation parameter \\
\hline$\xi$ & damping ratio \\
\hline$\theta_{k, \underline{\theta_{s}}}$ & stiffness and strength curve shape parameters, respectively \\
\hline$\varphi$ & frictional angle \\
\hline$\underline{\kappa} \underline{\theta} s$ & Pike's scale factor-curve shape parameter \\
\hline
\end{tabular}




\begin{tabular}{|l|l|}
\hline$\mu_{p}$ & Mass per unit length of the pile \\
\hline$\varphi$ & frictional angle \\
\hline
\end{tabular}




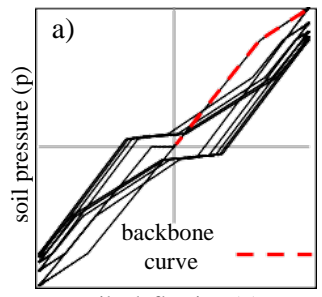

Pile deflection (y)

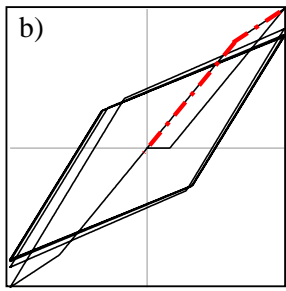

Pile deflection (y)

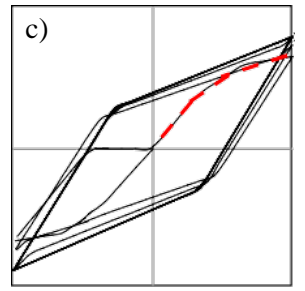

Pile deflection (y)

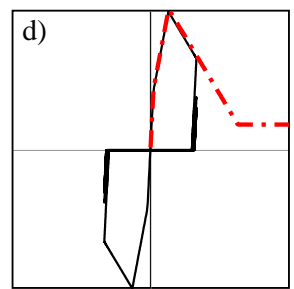

Pile deflection (y)

Figure 1. Hysteretic curves: a) S-shaped hysteresis curve; b) degrading oval-shaped curve, c)

hardening response and d) brittle behaviour. 
a)

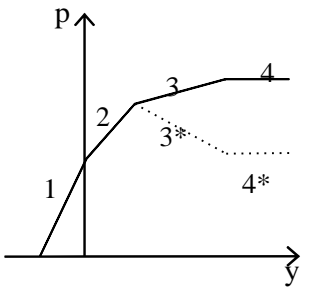

b)

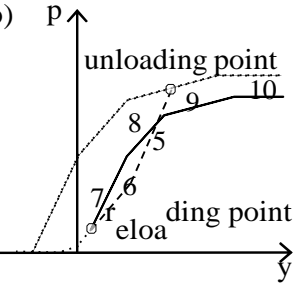

c)

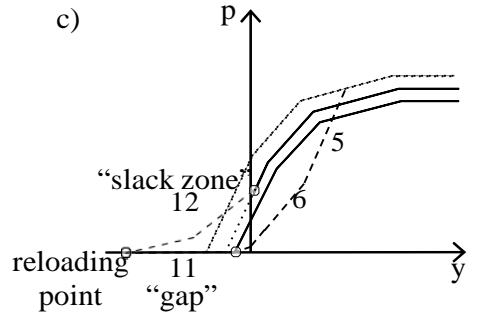

Figure 2. Cyclic p-y curves a) multilinear backbone curve; b) Standard Reload Curve and General

Unload Curve and c) Direct Reload Curve 

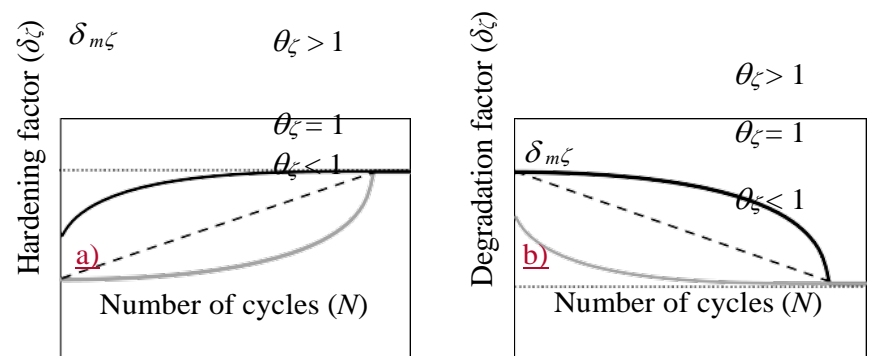

Figure 3. Elliptical a) hardening and b) /degradation curves for the stiffness degradation factor 
a)

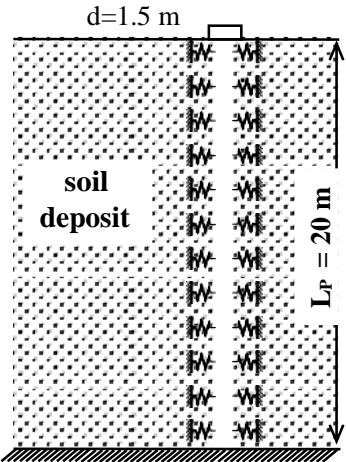

bedrock

$\mathrm{V}_{\mathrm{s}}=800 \mathrm{~m} / \mathrm{s}-\xi=5 \%$ b)

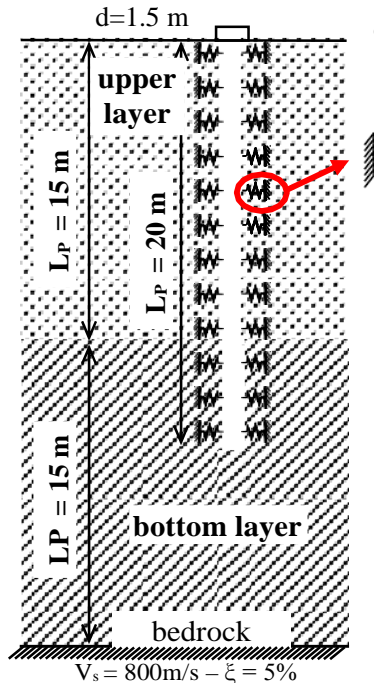

c) stiffness proportional damping

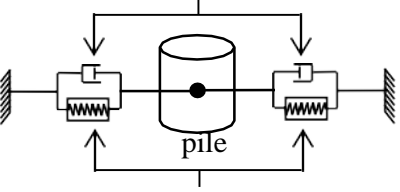

Nonlinear p-y curve:

- Pyke's rules

- gap

- "soil cave-in" effect

- hardening/degratation

Figure 4. a) Soil profile and b) pile modelled as an elastic beam supported

by non-linear uncoupled springs attached to each pile node in both sides 


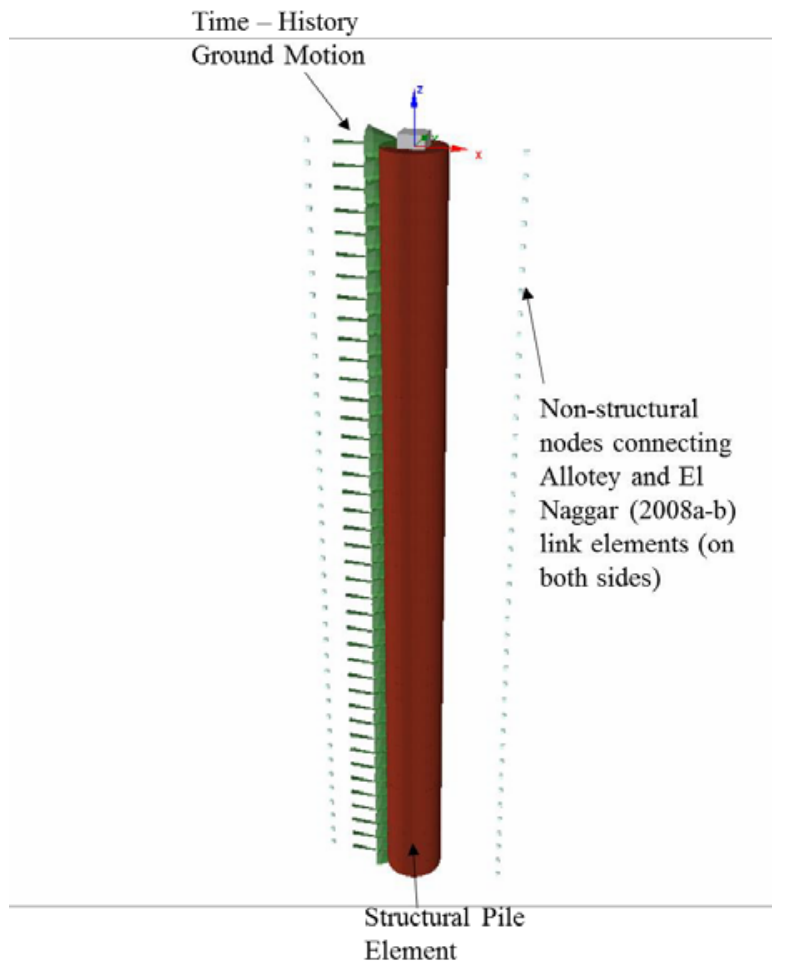

Figure 5. Finite Element Model realized in SeismoStruct (2013) comprised of monopile and link elements simulating the Allotey and El Naggar (2008a-b) degrading polygonal hysteretic model. Time-history ground motion accelerations are applied to each depth in order to simulate the soil wave propagation through the soil. 

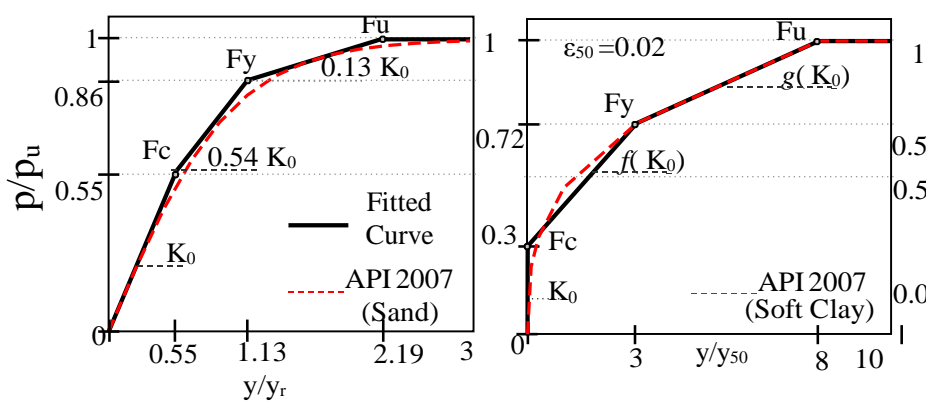

$\begin{aligned} & \text { Keese etal.1y/b } \\ & \text { (Stiff Clay) }\end{aligned}$
$\varepsilon_{50}=0.007$
$\mathrm{~g}\left(\mathrm{~K}_{0}\right)$
$\mathrm{y} / \mathrm{y}_{50} \quad \begin{aligned} & \text { U.09 } \\ & 10\end{aligned}$

Figure 6. Four-segment multi-linear fit to: (a) unit API p-y curve for sand; (b) unit API p-y curve for soft clay; (c) Reese et al. (1975) unit p-y curve for stiff clay. 
Table 1 Soil type and soil proprieties for homogeneous cases

\begin{tabular}{|l|l|l|l|l|l|l|l|l|l|}
\hline Soil & Soil & Soil & $\mathbf{D}_{\mathbf{r}}$ & $\mathbf{I}_{\mathbf{p}}$ & $\mathbf{V}_{\mathbf{s}}$ & $\boldsymbol{\gamma}$ & $\mathbf{v}$ & $\boldsymbol{\varphi}$ & $\mathbf{C}_{\mathbf{u}}$ \\
Deposit & Type & Consistency & {$[\%]$} & & {$[\mathbf{m} / \mathbf{s}]$} & {$\left[\mathbf{k N / \mathbf { m } ^ { 3 } ]}\right.$} & - & {$\left[{ }^{\circ}\right]$} & {$[\mathbf{k P a}]$} \\
\hline $\mathbf{1 0 0 D S}$ & Dry Sand & loose & 35 & $/$ & 100 & 14.22 & 0.3 & 30 & $/$ \\
$\mathbf{1 0 0 S S}$ & Saturated Sand & loose & 42 & $/$ & 100 & 19.65 & 0.3 & 33 & $/$ \\
$\mathbf{1 0 0 S C}$ & Saturated Clay & soft & $/$ & 27 & 100 & 15.5 & 0.45 & $/$ & 30 \\
$\mathbf{2 0 0 D S}$ & Dry Sand & medium dense & 55 & $/$ & 200 & 18.86 & 0.3 & 35 & $/$ \\
$\mathbf{2 0 0 S S}$ & Saturated Sand & medium dense & 63 & $/$ & 200 & 20.12 & 0.3 & 35 & $/$ \\
$\mathbf{2 0 0 S C}$ & Saturated Clay & medium & $/$ & 27 & 200 & 20.00 & 0.45 & $/$ & 75 \\
\hline
\end{tabular}


Table 2 Soil type and soil proprieties for bi-layer cases

\begin{tabular}{|l|l|l|l|l|l|l|l|l|l|}
\hline $\begin{array}{l}\text { Soil } \\
\text { Deposit }\end{array}$ & Soil & $\begin{array}{l}\text { Soil } \\
\text { Type }\end{array}$ & $\begin{array}{l}\text { Dr } \\
\text { Consistency }\end{array}$ & $\begin{array}{l}\text { Ip } \\
{[\mathbf{m} / \mathbf{s}]}\end{array}$ & $\begin{array}{c}\gamma \\
{\left[\mathbf{k N} / \mathbf{m}^{3}\right]}\end{array}$ & $\begin{array}{c}\boldsymbol{\varphi} \\
-\end{array}$ & $\begin{array}{l}\text { Cu } \\
{\left[{ }^{\circ}\right]}\end{array}$ & {$[\mathbf{k P a}]$} \\
\hline $\mathbf{1 0 0 3 0 0 D S}$ & Dry Sand & loose-dense & $\begin{array}{l}35- \\
70\end{array}$ & $/$ & $\begin{array}{l}100- \\
300\end{array}$ & 14.22 & 0.3 & $\begin{array}{l}30- \\
37\end{array}$ & $/$ \\
\hline $\mathbf{1 0 0 3 0 0 S S}$ & Saturated Sand & $\begin{array}{l}\text { Medium dense - } \\
\text { dense }\end{array}$ & $42-$ & $/$ & $\begin{array}{l}100- \\
3\end{array}$ & 19.65 & 0.3 & $\begin{array}{l}33- \\
37\end{array}$ & $/$ \\
\hline $\mathbf{1 0 0 3 0 0 S C}$ & Saturated Clay & Soft - stiff & $/$ & 27 & $\begin{array}{l}100- \\
300\end{array}$ & 20 & 0.4 & $/$ & $30-$ \\
\hline
\end{tabular}


Table 3 Cyclic and degradation model parameters for homogeneous cases

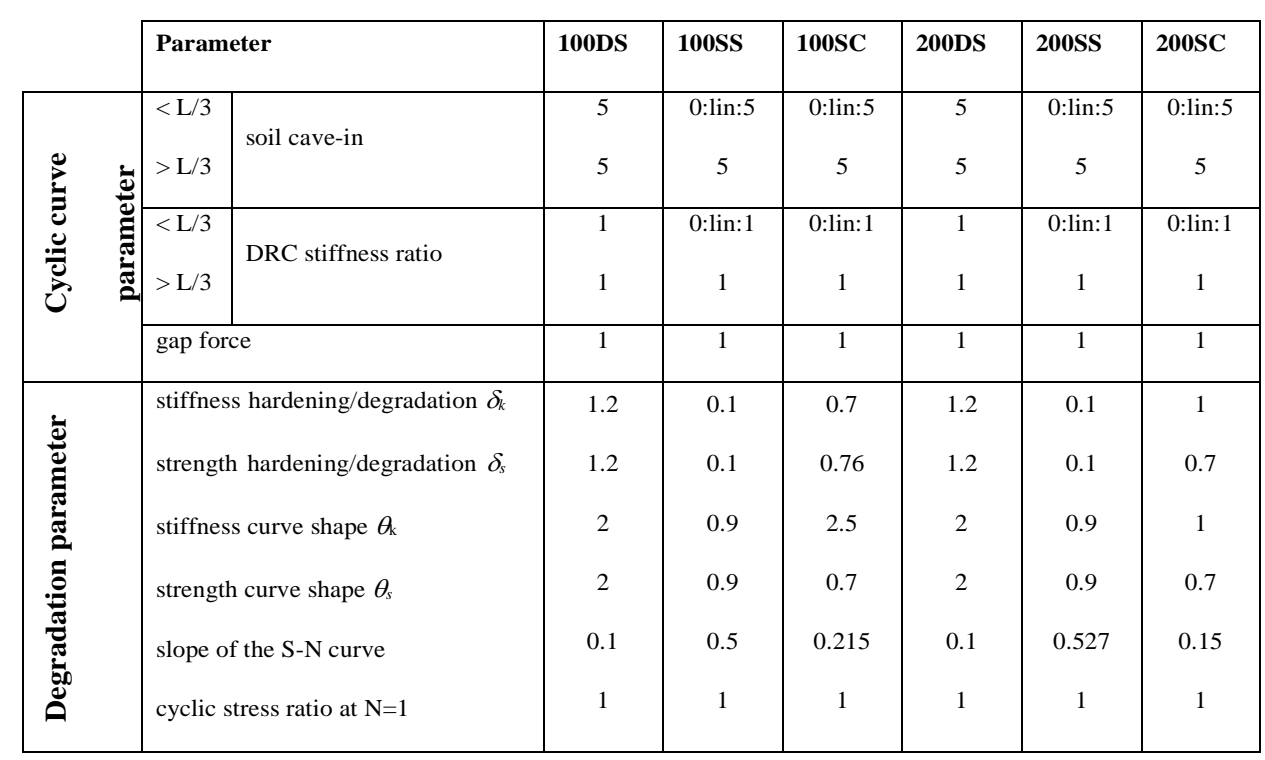


Table 4 Cyclic and degradation model parameters for bi-layer cases

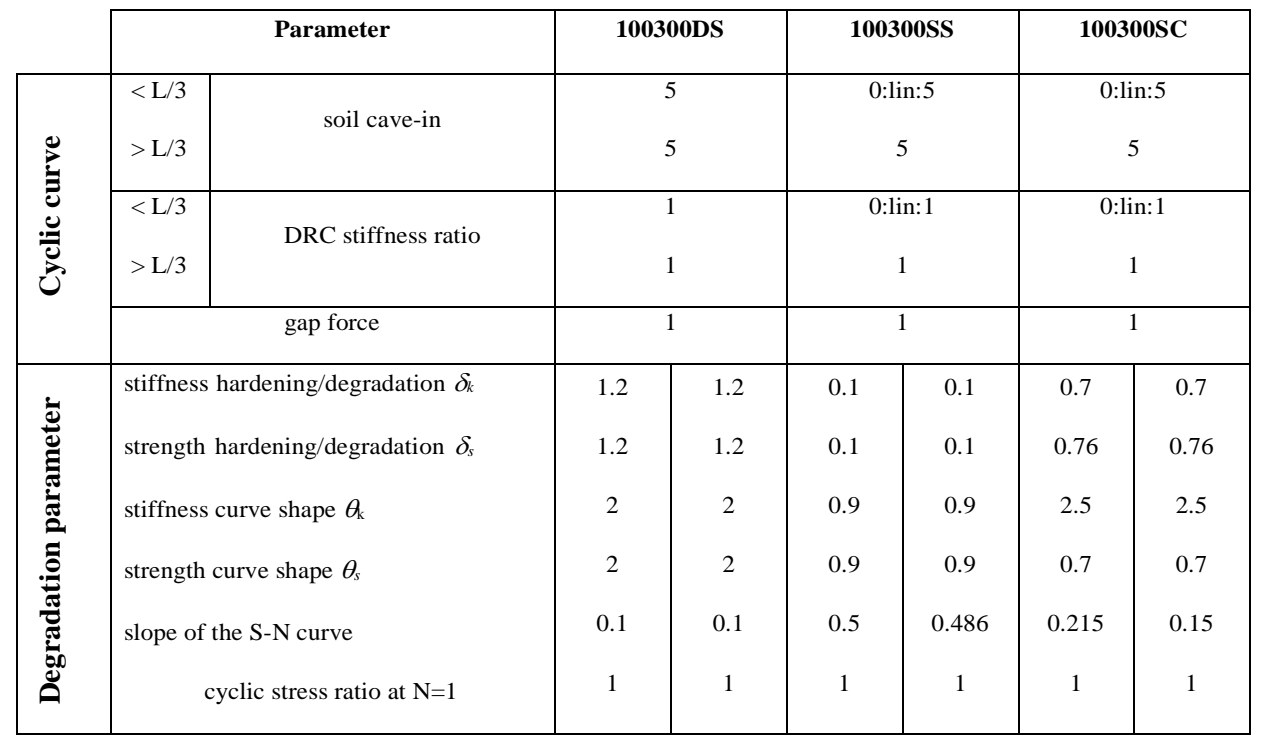




\begin{tabular}{|l|c|c|c|}
\hline EVENT & $\mathbf{I}_{\mathbf{d}}$ & $\mathbf{t}_{\mathbf{D}}$ & $\mathbf{t}_{\mathrm{E}}$ \\
{$[\mathrm{sec}]$} & {$[\mathrm{sec}]$} \\
\hline San Fernando & 4.4 & 7 & 9.22 \\
(09/02/1971) & 6.6 & 18 & 39.98 \\
Loma Prieta & 14.3 & 39 & 89.95 \\
Chi Chi & & & \\
(20/09/1999) & & & \\
Imperial Valley & 24.2 & 51 & 99.92 \\
\hline (15/10/1979) & & & \\
\hline
\end{tabular}

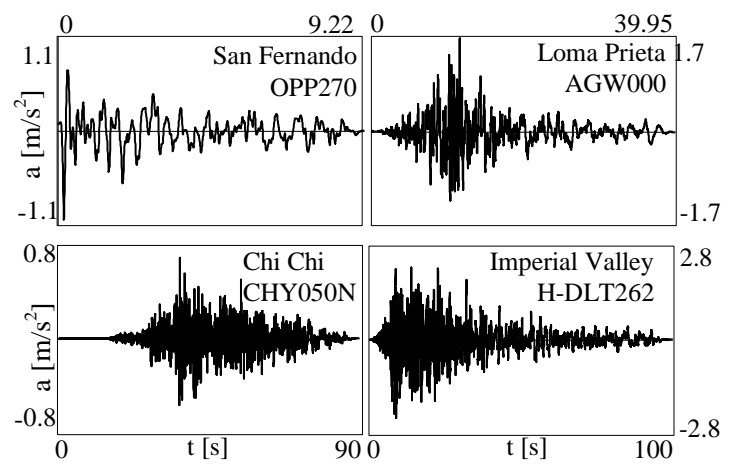

Figure 7. Earthquake records adopted in the analyses 


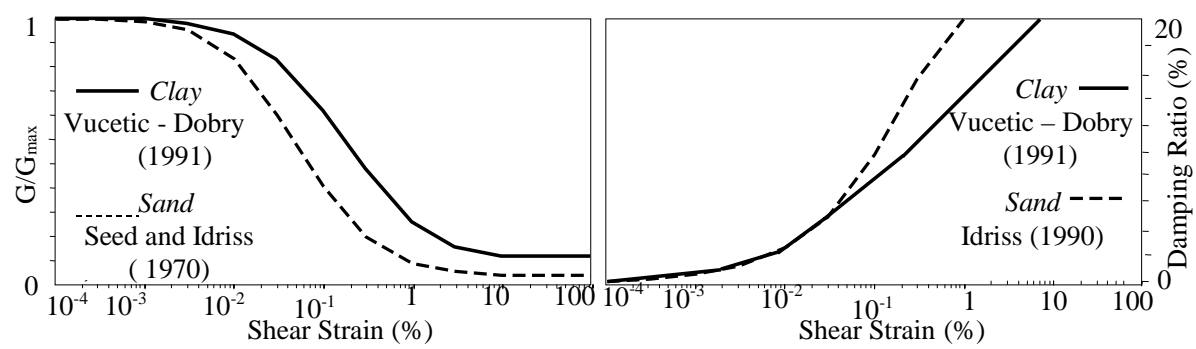

Figure 8. Variation of shear modulus and damping ratio with shear strain 
$\frac{\pi}{\pi}$

0

Figure 9. San Fernando earthquake: acceleration response spectra of the ground surface motion 


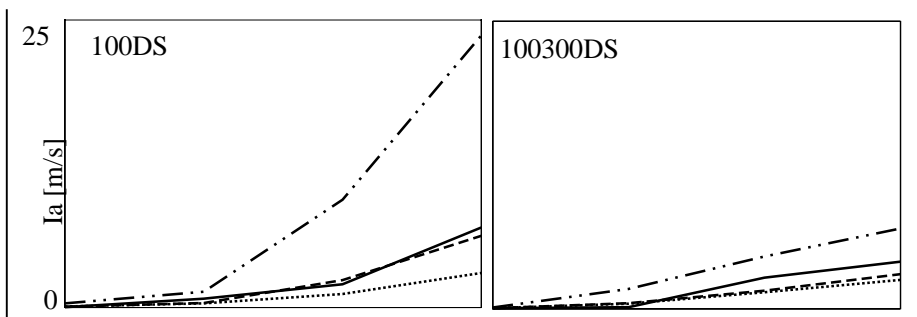

200DS

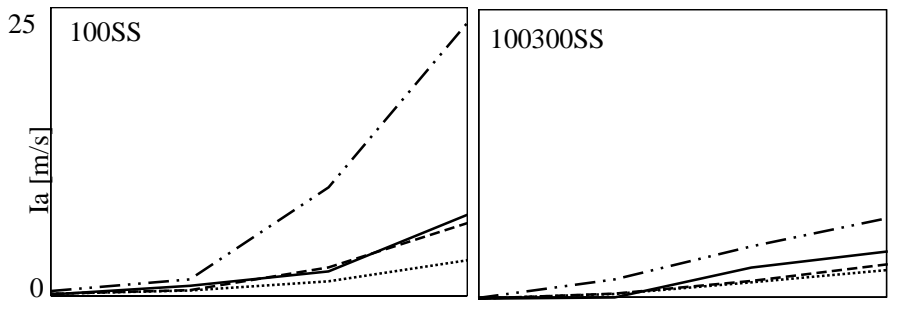

200SS

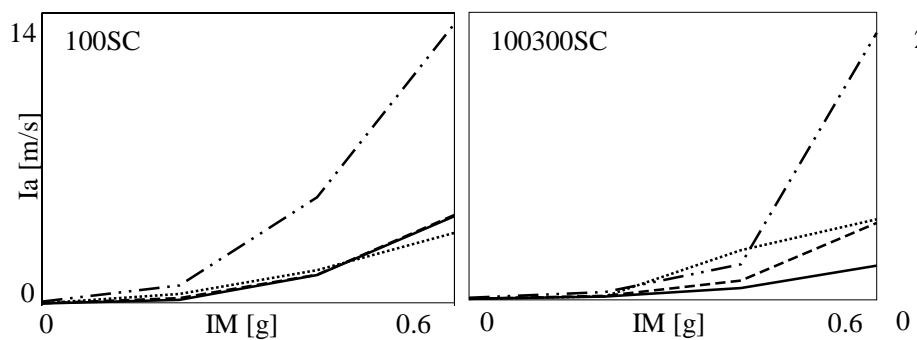

200SC

San Fernando

Loma Prieta

Chi Chi

Imperial Valley

[g]

0.6

Figure 910. Increment of the Arias Intensity of the ground-surface ground acceleration for the every investigated cases. 


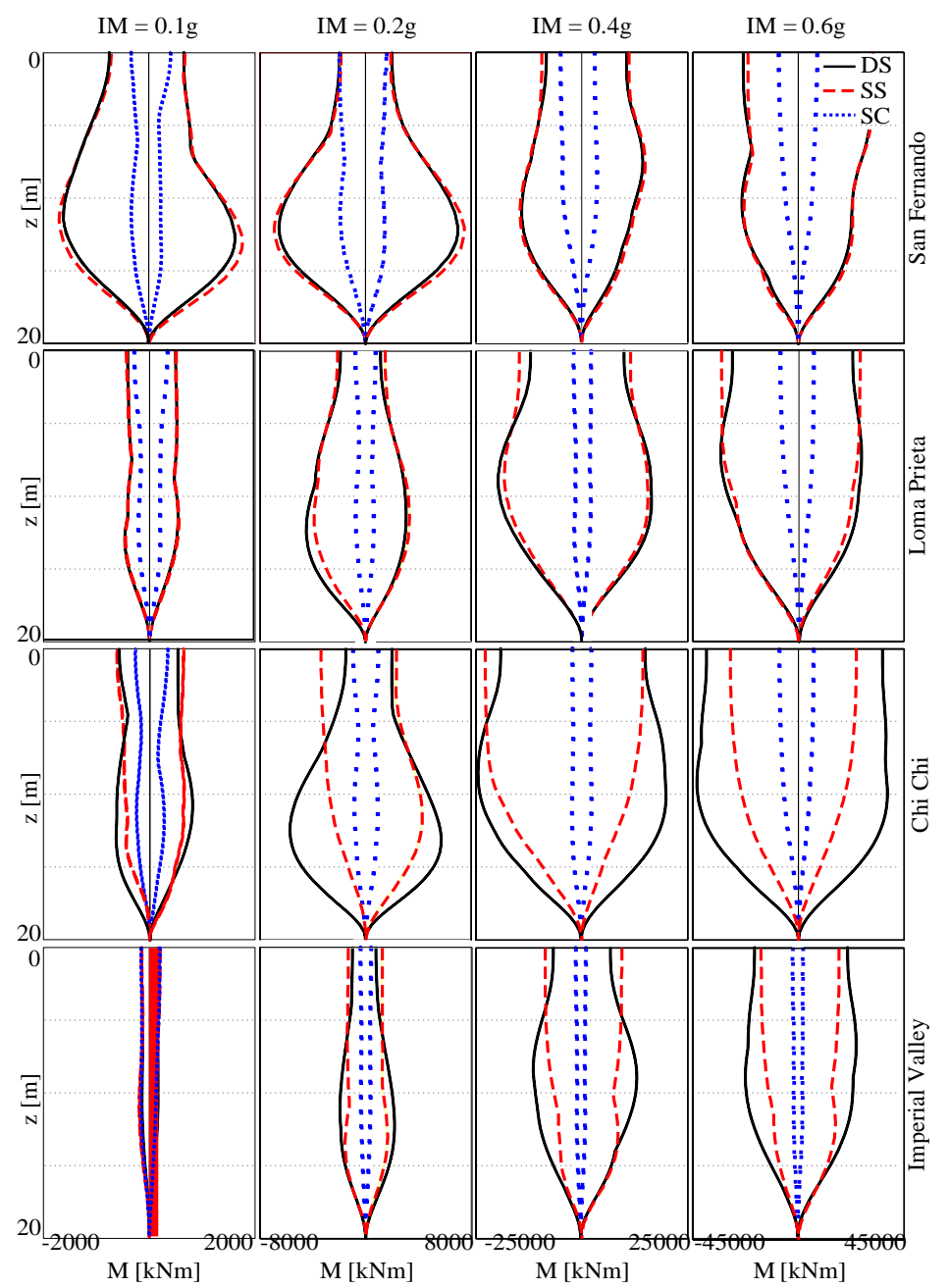

Figure 1011. Envelopes of bending moments obtained performing ADAs for soil profiles with Vs $=100 \mathrm{~m} / \mathrm{s}$. 


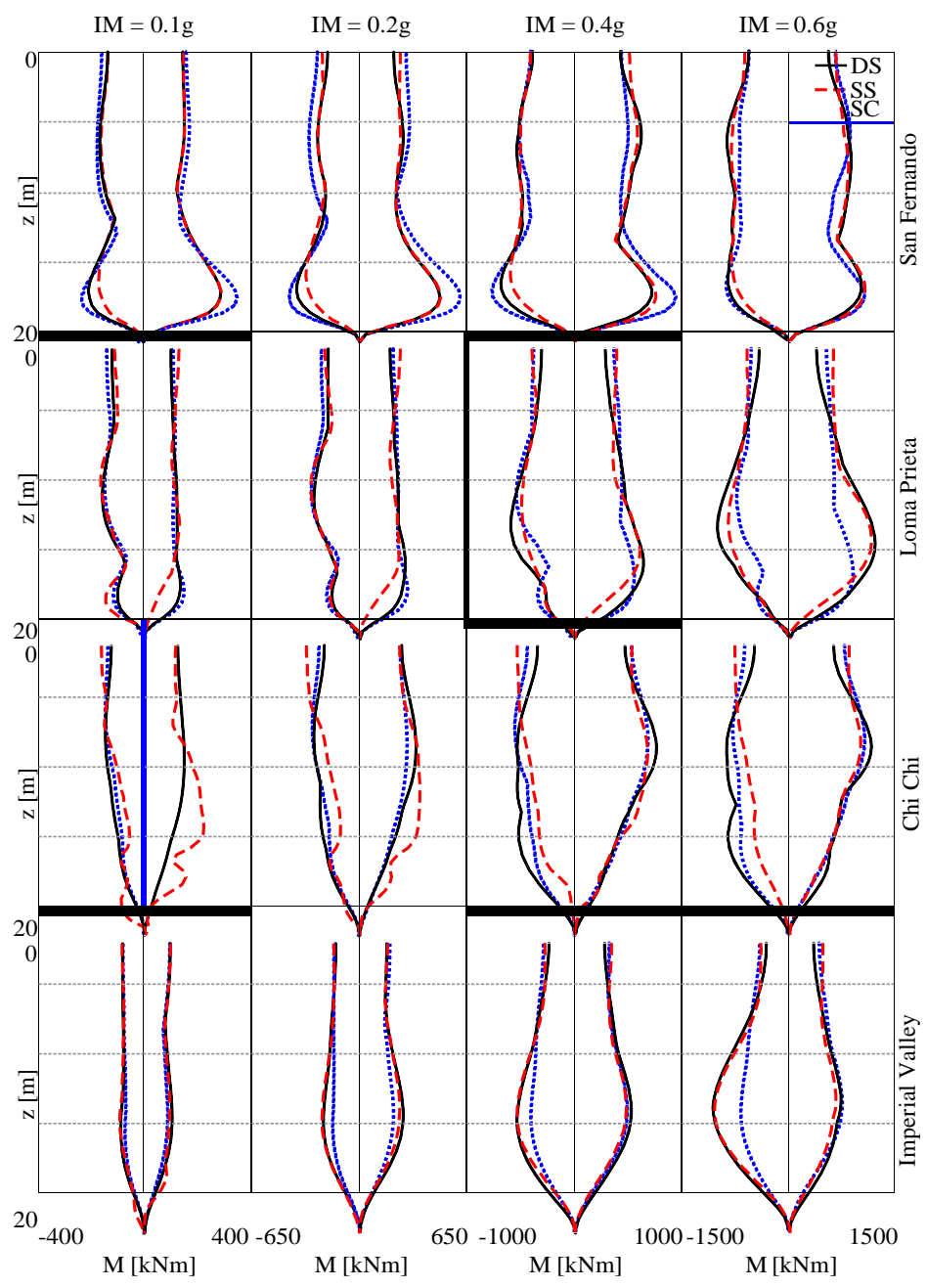

Figure $11 \underline{12}$. Envelopes of bending moments obtained performing IDAs for soil profiles with Vs $=\mathbf{2 0 0} \mathbf{~ m} / \mathrm{s}$. 


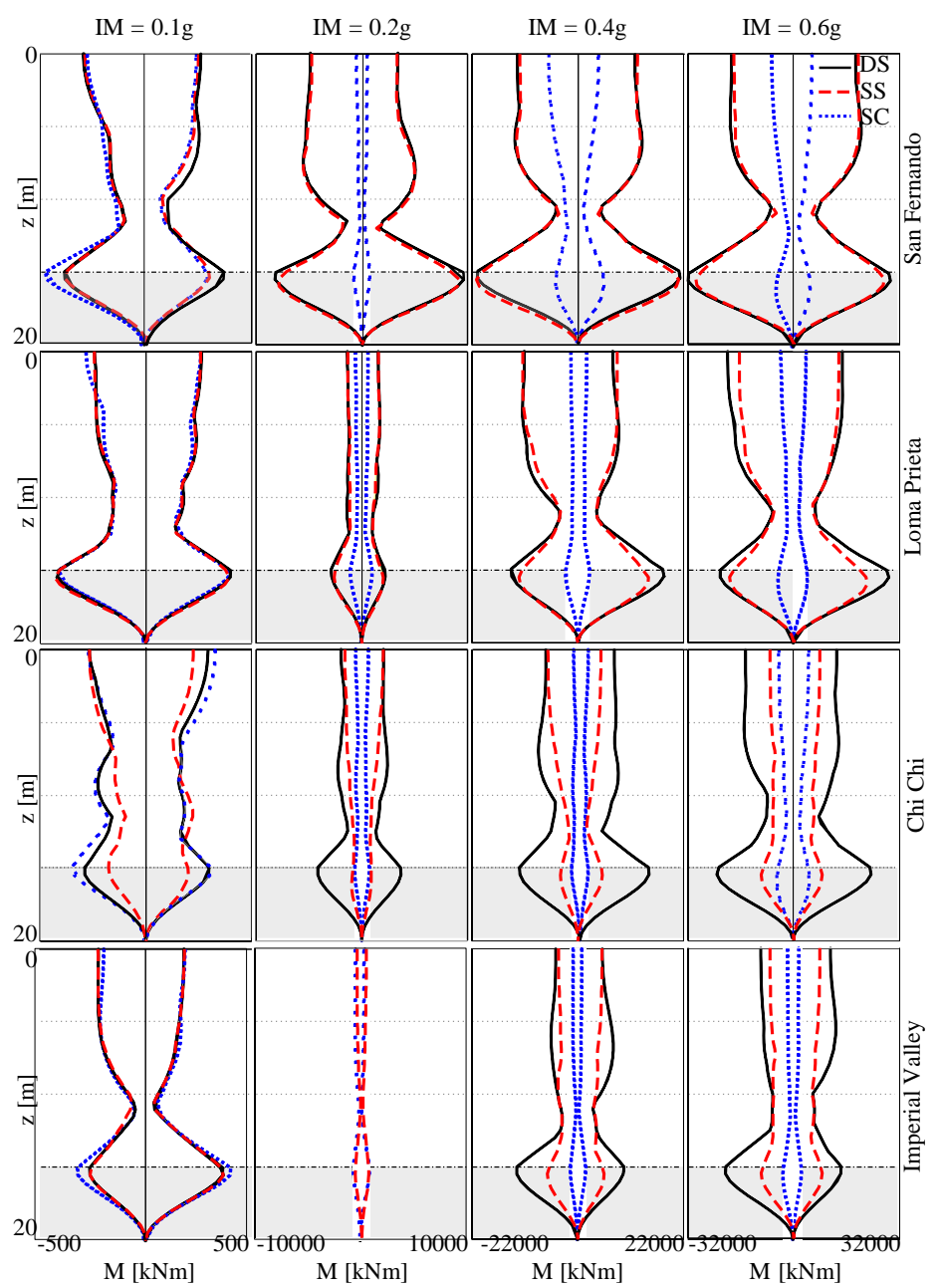

Figure $12 \underline{13}$. Envelopes of bending moments obtained performing IDAs for soil profiles with Vs = $100 \mathrm{~m}$ and Vs $=300 \mathrm{~m} / \mathrm{s}$ for the top and bottom layer, respectively 

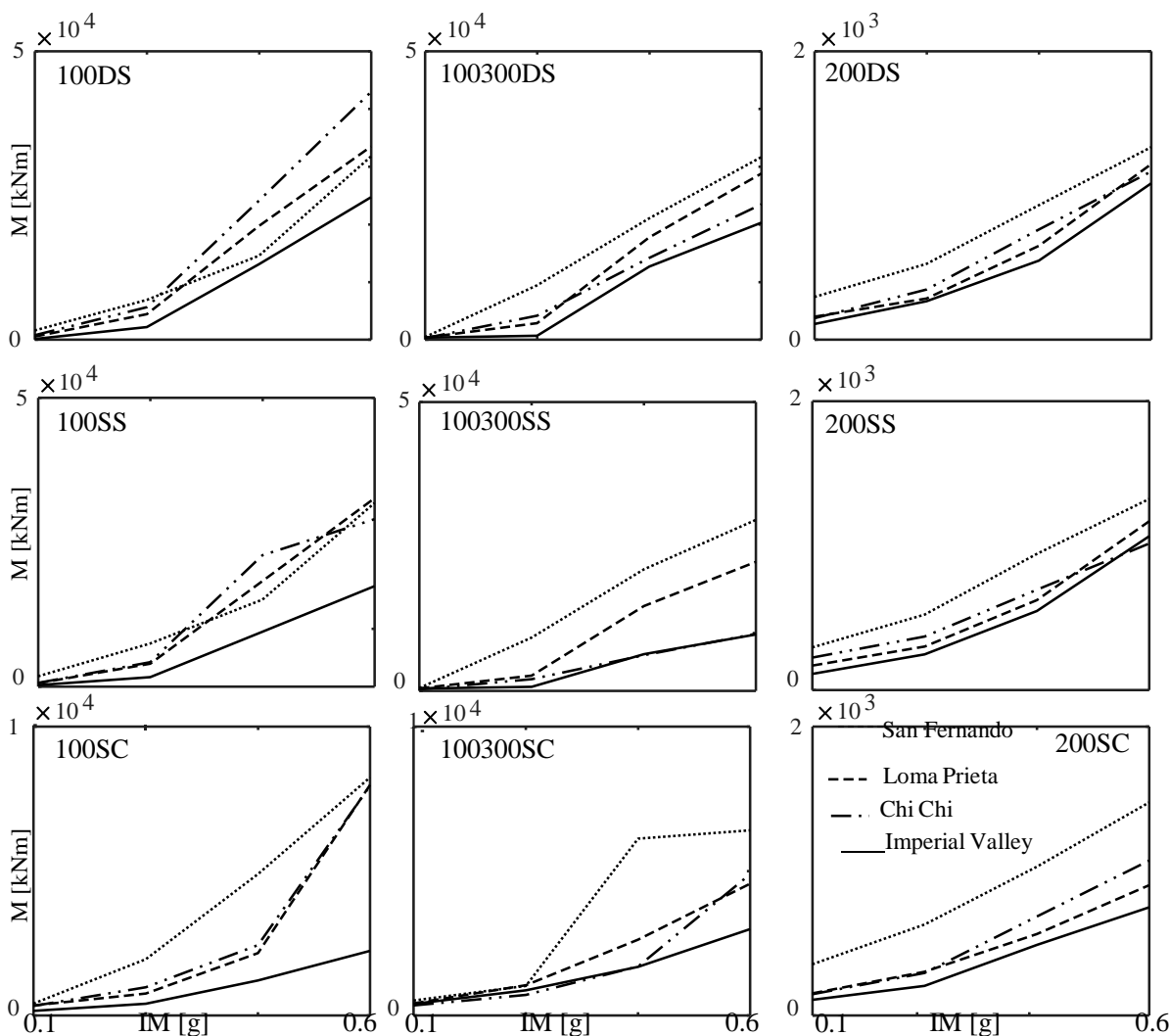

Figure 914. Increment of the maximum bending moment obtained XXXfor every investigated cases. 
0.12 100DS

100SS

100SC

$\frac{\Xi}{5}$

0

0.45 100300DS

100300SS

\begin{tabular}{l}
$\Xi$ \\
\hline
\end{tabular}

0

0.01 200DS 200SS

Imperial Valley
$\bar{\Xi}$

IM [g]

$0.6 \quad 0$

IM [g]

$0.6 \quad 0$

IM [g]

200SC

Loma Prieta

Chi Chi

Figure 915 . Increment of the foundation input motion in terms of maximum relative displacement for every 

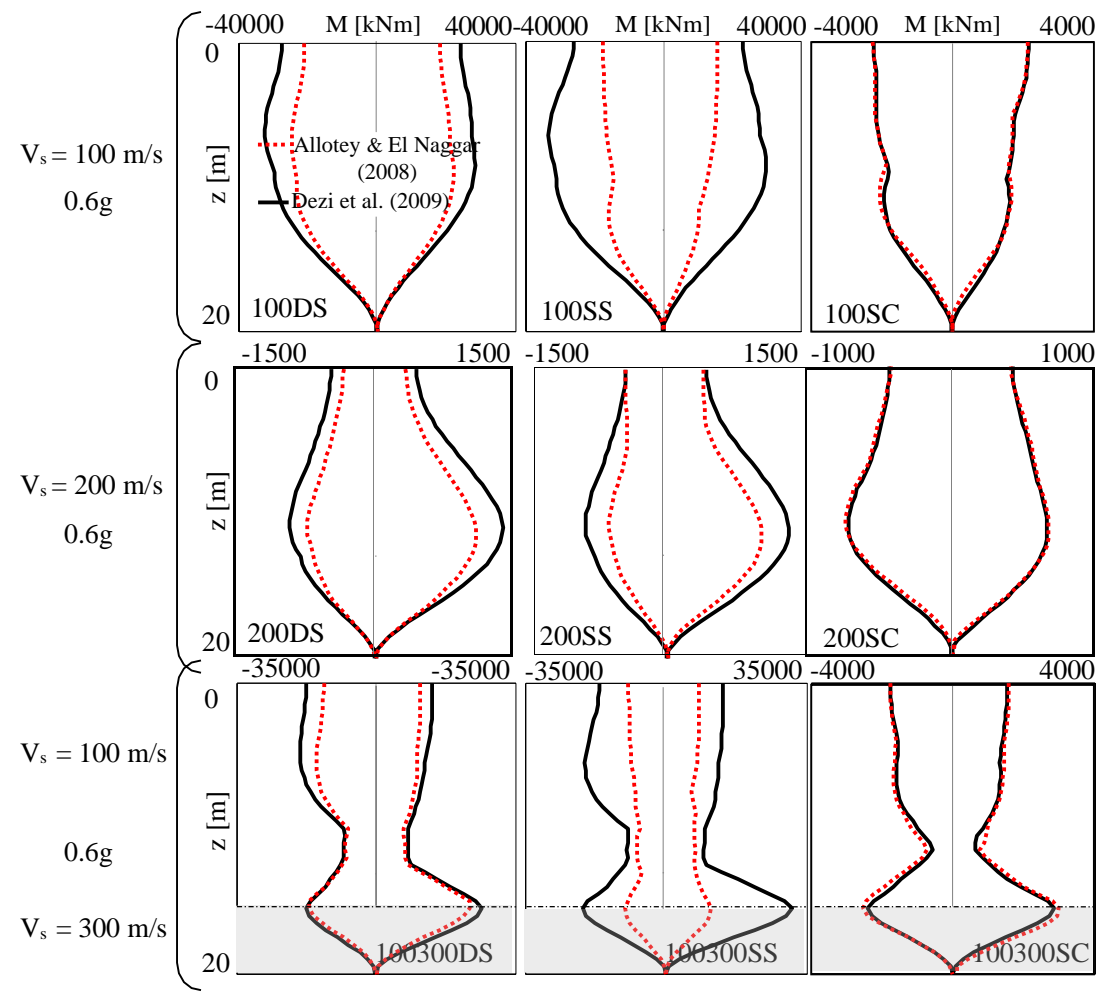

Figure 13-16 Bending moment envelopes obtained by considering a linear and nonlinear soil model for the Imperial Valley earthquake with $\mathrm{IM}=\mathbf{0 . 6 g}$. 

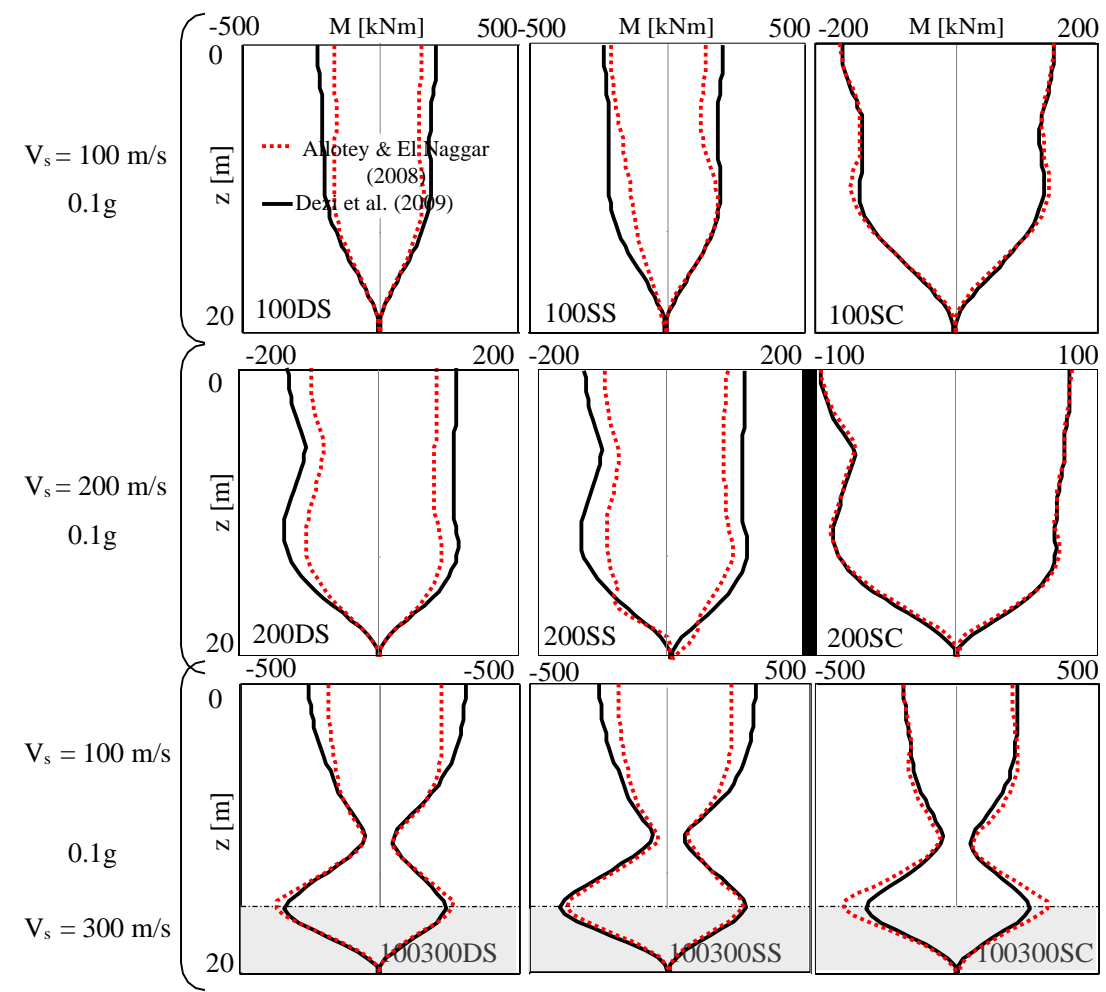

Figure 14-17 Bending moment envelopes obtained by considering a linear and nonlinear soil model for the

Imperial Valley earthquake with $\mathrm{IM}=\mathbf{0 . 1 \mathrm { g }}$. 